\title{
Superlinear Elliptic Equations and Systems
}

\author{
Bernhard Ruf \\ Dipartimento de Matematica \\ Università degli Studi di Milano \\ Via Saldini 50 \\ I-20133 Milano, Italy \\ ruf@mat.unimi.it
}

May 23, 2007

\section{Contents}

1. Introduction

2. Elliptic equations

2.1. Some history

2.2. My space or yours?

2.3. Compactness: the Sobolev embeddings

2.4. The phenomena of critical growth

3. The case of dimension $N=2$

3.1. Subcritical growth: the Trudinger-Moser inequality

3.2. Critical growth: a reslt of Carleson-Chang

3.3. Critical growth: existence of solutions

3.4. Critical growth: non-existence

4. Elliptic systems, $N \geq 3$

4.1. Strongly indefinite functionals

4.2. The critical hyperbola

4.3. The $H^{s}$-approach

4.3.1. Fractional Sobolev spaces and the functional setting

4.3.2. Compactness and existence of solutions for systems with subcritical growth

4.4. The $W^{1, \alpha}$ approach

4.4.1. The functional framework 
5. Generalization to Orlicz spaces

5.1. Orlicz spaces

5.2. Orlicz space criticality

5.3. Critical Orlicz-pairs: proof of Theorem 5.9

5.4. Orlicz space subcritical: an existence theorem

6. A system with no growth restriction in one nonlinearity

7. A borderline case

7.1. The $W^{s, \alpha}$-approach

7.1.1 Different results using the $H^{s}$-method and the $W^{s, \alpha}$-method

7.2. Lorentz spaces

7.3. Lorentz spaces and the asymptotic borderline case

7.4. Subcritical with respect to the asymptotic borderline case: existence of solution

8. Critical phenomena for the system

8.1. Critical growth: non existence of (positive) solutions

8.2. Critical growth: noncompactness and concentration

8.3. Critical growth: instantons

8.4. Brezis-Nirenberg type results for systems

9. Systems in two dimensions

9.1. Exponential Critical hyperbola

9.2. Existence for the subcritical problem in dimension $N=2$

9.3. Critical systems in dimension $N=2$ 


\section{Introduction}

In this article we survey some recent results on superlinear elliptic equations and systems. A particular focus will be the borderline situations of so-called critical growth. In the existence theorems, we will use mostly variational methods, that is we look for critical points of functionals associated to the equations and systems. In dealing with variational problems, the choice of the function spaces in which the functionals are defined is of essential importance. There are two competing factors which determine this choice: on the one hand, the space should be "sufficiently small" so that the functional under consideration has the desired regularity; and on the other hand, the space should "not be too small" since otherwise the required compactness properties may get lost.

When working with scalar equations one is used that the above smallness and largeness requirements usually lead to a unique choice of (Sobolev) space, in which the problem is well-posed and hence solvable. Indeed, the borderline situation (critical growth) may be defined as the limiting situation in which this space setup works. We will discuss the various phenomena connected with critical growth.

We then treat recent results on systems of superlinear equations. We will see that for the functionals associated to systems there is more freedom in the choice of the space; in fact, one may choose among a whole continuum of products of Sobolev spaces. Each choice yields different maximal growths for the respective nonlinearities, but again we find that for a fixed pair of such critical growth nonlinearities there exists a unique choice of a product Sobolev space. Together the pairs of critical growth nonlinearities form the so-called "critical hyperbola".

We will then concentrate on some limiting cases of elliptic systems. Contrary to the situation in scalar equations and in (non limiting case) systems, we will find a wide range of (Sobolev) spaces available in which the corresponding functionals may be defined, and the question of the "right" functional setup becomes quite delicate. Indeed, we will see that in some limiting cases the various possible choices of Sobolev spaces yield, for the same functional, different maximal growths. We will then see that the more refined Sobolev-Lorentz spaces provide an "optimal" functional setup.

Much space will be devoted to the less widely known situation in dimension $N=2$, where critical growth is of exponential type, given by the so-called Trudinger-Moser inequality. Working with systems in dimension $N=2$, we will see that also here the Sobolev-Lorentz spaces yield the suitable functional setup in which the analogue of the critical hyperbola (involving nonlinearities of different exponential growths) can be found. 


\section{Elliptic equations}

\subsection{Some history}

For studying the model elliptic equation

$$
\left\{\begin{array}{clrl}
-\Delta u & =f(u) & & \text { in } \Omega \\
u & =0 & & \text { on } \partial \Omega,
\end{array}\right.
$$

where $\Omega \subset \mathbb{R}^{n}$ is an bounded open domain and $f: \mathbb{R} \rightarrow \mathbb{R}$ is a continuous function, one may try to use the well-known Dirichlet principle which consists in minimizing an associated functional over a suitable set of functions; then, the corresponding critical points correspond by the Euler-Lagrange principle to (weak) solutions of problem (2.1). The functional associated to equation (2.1) takes the form

$$
I(u)=\frac{1}{2} \int_{\Omega}|\nabla u|^{2} d x-\int_{\Omega} F(u) d x
$$

where $F(t)=\int_{0}^{t} f(s) d x$ is the primitive of $f(t)$.

If we for instance assume that

$$
|f(s)| \leq M, \forall s \in \mathbb{R}
$$

and hence

$$
|F(s)| \leq c+M|s|, \forall s \in \mathbb{R}
$$

we can estimate

$$
\begin{aligned}
& I(u) \geq \frac{1}{2} \int_{\Omega}|\nabla u|^{2} d x-M \int_{\Omega}|u| d x \\
& \geq \frac{1}{2} \int_{\Omega}|\nabla u|^{2} d x-M|\Omega|^{1 / 2}\left(\int_{\Omega}|u|^{2} d x\right)^{1 / 2}
\end{aligned}
$$

By the Poincaré inequality

$$
\int_{\Omega}|\nabla u|^{2} \geq d \int_{\Omega}|u|^{2}
$$

we find that $I(u)$ is bounded from below, and hence is makes sense to look for the global minimum of this functional. It is clear that there exist functionals which are bounded below but which do not attain their minimum: consider e.g. $j: \mathbb{R} \rightarrow \mathbb{R}: j(s)=e^{s}$; clearly, $\inf _{s \in \mathbb{R}} j(s)=0$, and any minimizing sequence $\left(s_{n}\right)$ satisfies $s_{n} \rightarrow-\infty$. The above functional $I(u)$ seems better behaved since we see easily that any minimizing sequence $\left(u_{n}\right) \subset H_{0}^{1}(\Omega)$ is in fact bounded: Setting $m=\inf _{u \in H_{0}^{1}(\Omega)} I\left(u_{n}\right)$, we have

$$
m+1 \geq I\left(u_{n}\right) \geq \frac{1}{2} \int_{\Omega}\left|\nabla u_{n}\right|^{2} d x-M|\Omega|^{1 / 2} d\left(\int_{\Omega}\left|\nabla u_{n}\right|^{2} d x\right)^{1 / 2}
$$


and hence

$$
c \geq\left(\int_{\Omega}\left|\nabla u_{n}\right|^{2} d x\right)^{1 / 2}=\left\|u_{n}\right\|_{H_{0}^{1}}
$$

In finite dimensions this would lead immediately to a convergent subsequence and to the conclusion that the minimum is attained. However, in the infinite dimensional space $H_{0}^{1}(\Omega)$ the situation is more delicate. Indeed, in Dirichlet's time, and also much later, this questions was not rigorously posed, and it was often tacitly assumed that the minima for functionals of the form $I(u)$ are attained, without questioning by what kind of function. It took the famous Weierstrass example, namely

$$
J(u)=\int_{0}^{1} x^{2}\left|u^{\prime}\right|^{2} d x, u:[0,1] \rightarrow \mathbb{R}, u(0)=0, u(1)=1
$$

to change things. Minimizing $J(u)$ for instance over the (natural) class $E=$ $\left\{u \in C^{1}([0,1], \mathbb{R}) ; u(0)=0, u(1)=1\right\}$, one sees easily that inf $J(u)=0$, but that 0 cannot be attained by a $C^{1}$-function. This threw the field of Calculus of Variations (and in fact all of Analysis) into a crisis; but the crisis was overcome by the efforts of Weierstrass himself, by Arzelà, Fréchet, Hilbert, Lebesgues and others, leading to the foundation of modern analysis.

\subsection{My space or yours?}

In today's words, the upshot from this crisis regarding the Dirichlet principle is precisely the question over what class (or space) of functions the minimization should be taken. Indeed, there is a large variety of spaces available, the spaces of continuous and differentiable functions, the more refined Hölder spaces, the more general Lebesgues and Sobolev spaces, and (as we will see later on) generalizations of these, the Orlicz spaces and Lorentz spaces. So the actual choice of the space to work in seems somewhat arbitrary - and only restricted by the expectation that the "outcome" should be (essentially) the same, and not really depend on the choice of the space. This apparent ambiguity in the choice of the space may be hard to understand for people working in other fields - it has even been said that: "if the space matters, then it doesn't matter ..."

There are two competing requirements which intervene in the choice of the space: the functional must be continuous and differentiable, and the functional must possess a suitable compactness; for the first requirement, the space should be "small", i.e. the topology must be sufficiently fine. Indeed, if we take the "small" space of differentiable $C^{1}$-functions, then the functional of the form

$$
I(u)=\frac{1}{2} \int_{\Omega}|\nabla u|^{2} d x-\int_{\Omega} F(u) d x
$$

is certainly defined and continuous in $u$; however, due to the incompleteness of this space with respect to the Dirichlet-norm $\|u\|=\left(\int_{\Omega}|\nabla u|^{2} d x\right)^{1 / 2}$, 
compactness fails to hold, and it is in general impossible to prove directly that in $f_{C^{1}(\Omega)} I(u)$ is attained. On the other hand, for the compactness requirement, the space should be sufficiently "large", i.e. the coarser the topology the better.

Indeed, by Sobolev's work we know that a "good" space to work with is the Sobolev space $H_{0}^{1}(\Omega)$, i.e. the space of functions whose (weak) derivative belongs to the space $L^{2}(\Omega)$. By the very definition of this space, the Dirichlet integral is well-defined and continuous on this space; in fact, it is the largest space on which this is the case. Now, we need to check that also the second part of the functional $I(u)$, i.e. $\int_{\Omega} F(u) d x$, is well-defined on $H_{0}^{1}(\Omega)$. To obtain this, one needs to impose a growth condition on $f(s)$, namely

$$
|f(s)| \leq c+c|s|^{\frac{n+2}{n-2}}, s \in \mathbb{R}
$$

This implies that $F(s)=\int_{0}^{s} f(t) d t$ satisfies the restriction

$$
|F(s)| \leq c+c|s|^{2^{*}}, s \in \mathbb{R}, 2^{*}:=\frac{2 N}{N-2}, N \geq 3 .
$$

And then, by the famous Sobolev embedding theorem $H_{0}^{1}(\Omega) \subset L^{2^{*}}(\Omega)$, one concludes that indeed the second term of the functional $I(u)$ is well-defined on $H_{0}^{1}(\Omega)$, and that the functional is continuous and differentiable.

\subsection{Compactness}

Let us now return to the question raised above, namely whether it is true that "bounded minimizing sequences contain a convergent subsequence". In the context of more general critical points, this is known today as the Palais-Smale property, and goes back to a famous work [39] of these two authors where they study a generalized Morse theory.

Indeed, if we impose the stronger growth condition (so-called subcritical growth):

$$
|f(s)| \leq c_{1}+c_{2}|s|^{p}, \quad \text { for some } 1<p<\frac{N+2}{N-2},
$$

then we have by Rellich's theorem a compact embedding $H_{0}^{1}\left(\Omega \subset L^{p+1}(\Omega)\right.$, and consequently the Palais-Smale property is often (that is, under suitable "technical" conditions) satisfied.

On the other hand, if we consider the model problem ${ }^{1}$ with "critical growth"

$$
\left\{\begin{array}{l}
-\Delta u=u^{\frac{N+2}{N-2}}, \text { in } \Omega \\
u(0)=0, \text { on } \partial \Omega
\end{array},\right.
$$

then interesting phenomena appear: first of all, by the famous Pohozaev identity [40], one proves that equation (2.2) has no nontrivial solution if $\Omega$ is starshaped. Thus, one might argue that the problem is completely solved:

\footnotetext{
${ }^{1}$ to simplify notation, we will write throughout the text: $s^{p}:=|s|^{p-1} s$
} 
- the (in some sense natural) choice of the space $H_{0}^{1}(\Omega)$ yields compactness and hence solvability for subcritical growth (defined precisely by this choice of the space);

- on the other hand, the Palais-Smale property fails and solvability is lost at the limiting "critical growth".

But due to the continued interest in equations of form (2.2), mainly because of their importance in geometry, the studies continued, and many surprising and fascinating phenomena were discovered.

\subsection{Critical growth}

In this section we discuss some of the main phenomena of critical growth:

\section{Loss of compactness:}

The "loss of compactness" derives from the non compactness of the limiting case of the Sobolev embedding

$$
H_{0}^{1}(\Omega) \subset L^{2^{*}}(\Omega), 2^{*}=\frac{2 N}{N-2}, N \geq 3 .
$$

To see that this embedding is not compact, it suffices to find a bounded sequence $\left(u_{n}\right) \subset H_{0}^{1}(\Omega)$ which does not admit a convergent subsequence in $L^{2^{*}}(\Omega)$. Such a sequence can be easily constructed: Choose a ball of radius $a$ such that $B_{a}\left(x_{0}\right) \subset \Omega$. Clearly we may assume that $x_{0}=0$. Let

$$
u_{n}(x)=u_{n}(|x|)=u_{n}(r)= \begin{cases}\frac{1}{n^{\frac{N-2}{2}}} \frac{1}{r^{N-2}}-d_{n} & , \frac{1}{n} \leq r \leq a \\ n^{\frac{N-2}{2}}-d_{n} & , 0 \leq r \leq \frac{1}{n}\end{cases}
$$

where $d_{n}=\frac{1}{n^{\frac{N-2}{2}} a^{N-2}}$, and $u_{n}(x)=0$ for $x \in \Omega \backslash B_{a}(0)$.

A direct calculation shows that the Dirichlet norm $\|u\|=\left(\int_{\Omega}|\nabla u|^{2} d x\right)^{1 / 2}$ is bounded: indeed

$$
\int_{\Omega}\left|\nabla u_{n}\right|^{2} d x=\omega_{N-1} \frac{(N-2)^{2}}{n^{N-2}} \int_{1 / n}^{a} r^{2-2 N} r^{N-1} d r=C_{N}\left(1-\frac{c(a)}{n^{N-2}}\right) \leq C_{N},
$$

where $\omega_{N-1}$ denotes the surface area of the unit sphere in $\mathbb{R}^{N}$.

Furthermore, we observe that pointwise $u_{n}(r) \rightarrow 0$, for all $0<r<a$, as $n \rightarrow \infty$. But $\left(u_{n}\right)$ cannot have a subsequence which converges to 0 in $L^{2^{*}}(\Omega)$, since

$$
\int_{\Omega}\left|u_{n}\right|^{2^{*}} d x \geq \omega_{N-1} \int_{0}^{1 / n}\left(n^{\frac{N-2}{2}}-d_{n}\right)^{\frac{2 N}{N-2}} r^{N-1} d r \geq c_{1}>0, \forall n \in \mathbb{N} .
$$


From the simple sequence above, we may make another very important observation. Looking at the "principal term" of $u_{n}$, namely,

$$
\tilde{u}_{n}(r):=\frac{1}{n^{\frac{N-2}{2}}} \frac{1}{r^{N-2}}=n^{\frac{N-2}{2}} \frac{1}{(n r)^{N-2}}
$$

we observe that

$$
\tilde{u}_{n}(r)=n^{\frac{N-2}{2}} \tilde{u}_{1}(n r)
$$

and from the simple calculations above we see that $\int_{\Omega}\left|\nabla \tilde{u}_{1}\right|^{2} d x$ as well as $\int_{\Omega}\left|\tilde{u}_{1}\right|^{2^{*}} d x$ remain invariant under this "group action". This is in fact true in general, i.e. in the limiting Sobolev embedding we have a

\section{Group invariance:}

For $u \in H_{0}^{1}(\Omega)$, define the continuous group action or "rescaling"

$$
u_{\lambda}(x):=\lambda^{\frac{N-2}{2}} u(\lambda x) ;
$$

this is defined for all $\lambda>0$ if $\Omega=\mathbb{R}^{N}$, and for $0<\lambda \leq 1$ if $\Omega$ is starshaped (w.r.t. the origin). One then shows by direct calculation that

$$
\int_{\Omega}\left|\nabla u_{\lambda}\right|^{2} d x=c, \int_{\Omega}\left|u_{\lambda}\right|^{2^{*}} d x=d, \forall \lambda>0
$$

It is in fact the appearance of this invariance under rescaling which is the deeper reason for the loss of compactness.

\section{Non-existence of solutions in bounded starshaped domains:}

With the loss of compactness, one loses the main instrument to prove existence of a solution to equation (2.2). And in fact, one may show that if $\Omega$ is a bounded starshaped domain, then indeed there does not exist a (non trivial) solution.

This is due to the famous Pohozaev identity, see [40]. This identity is obtained from the equation (2.2) by multiplication by $x \cdot \nabla u$ and integration, and it says that if $u \in C^{2}(\Omega) \cap C^{1}(\bar{\Omega})$ is a solution of $(2.2)$, then the following relation holds:

$$
\frac{N-2}{2} \int_{\Omega}|\nabla u|^{2} d x-\frac{N}{2^{*}} \int_{\Omega}|u|^{2^{*}} d x+\frac{1}{2} \int_{\partial \Omega}\left|\partial_{\nu} u\right|^{2} x \cdot \nu d \sigma=0,
$$

where $\nu$ is the exterior normal to $\partial \Omega$. On the other hand, multiplying (2.2) by $u$ and integration yields

$$
\int_{\Omega}|\nabla u|^{2} d x=\int_{\Omega}|u|^{2^{*}} d x .
$$

From these two identities follows that

$$
\int_{\partial \Omega}\left|\partial_{\nu} u\right|^{2} x \cdot \nu d \sigma=0,
$$


Since $\Omega$ is starshaped, we have $x \cdot \nu>0$, for all $x \in \partial \Omega$, and hence $\partial_{\nu} u=0$ on $\partial \Omega$. But this implies that $u \equiv 0$ by the principle of unique continuation.

The situation changes if we consider the problem on the whole of $\mathbb{R}^{N}$. In the case with critical growth we have:

\section{Existence of explicit solutions on $\mathbb{R}^{n}$ : instantons}

The explicit solutions of the equation

$$
\left\{\begin{array}{l}
-\Delta u=u^{\frac{n+2}{n-2}}, \text { in } \mathbb{R}^{N} \\
u(0) \rightarrow 0, \text { for }|x| \rightarrow 0
\end{array}\right.
$$

are

$$
u_{\lambda}(x)=(N(N-2))^{\frac{N-2}{4}} \frac{\lambda^{\frac{N-2}{2}}}{\left(\lambda^{2}+|x|^{2}\right)^{\frac{N-2}{2}}}, \lambda>0 .
$$

They were found independently by G. Talenti [48] and Th. Aubin [7]. Note that (2.5) represent a family of solutions, parametrized by $\lambda>0$. This reflects again the group (or scaling) invariance of the equation. In addition, there is also (an obvious) invariance of the equation by translation. One knows that, up to the rescaling and translations, the above solutions are the only solutions of equation (2.4). The solutions are characterized by

$$
S_{N}\left(\mathbb{R}^{N}\right)=\inf _{u \in H^{1}\left(\mathbb{R}^{N}\right) \backslash\{0\}} \frac{\int_{\mathbb{R}^{N}}|\nabla u|^{2} d x}{\left(\int_{\mathbb{R}^{N}}|u|^{2^{*}} d x\right)^{2 / 2^{*}}}
$$

This expression characterizes the best Sobolev embedding constant for the embedding $H^{1}\left(\mathbb{R}^{N}\right) \subset L^{2^{*}}\left(\mathbb{R}^{N}\right)$, and thus this constant is attained by the function (2.5), and it can be explitely calculated. On the other hand, by the Pohozaev identity we infer that the best Sobolev embedding constant $S_{N}(\Omega)$ cannot be attained if $\Omega$ is starshaped: otherwise, we would obtain a nontrivial solution to equation (2.4) which is impossible. In fact, by the unique continuation property, one shows that this constant is never attained if $\Omega \neq \mathbb{R}^{N}$.

We have alrady seen that there is a loss of compactness for the embedding $H_{0}^{1}(\Omega) \subset L^{2^{*}}(\Omega)$, for bounded domains $\Omega \subset \mathbb{R}^{n}$, by giving an explicit bounded sequence in $H_{0}^{1}(\Omega)$ which does not have a convergent subsequence in $L^{2^{*}}(\Omega)$. Using the instantons (2.5), is is easy to obtain a minimizing sequence for

$$
S_{N}(\Omega)=\inf _{u \in H_{0}^{1}(\Omega) \backslash\{0\}} \frac{\int_{\Omega}|\nabla u|^{2} d x}{\left(\int_{\Omega}|u|^{2^{*}} d x\right)^{2 / 2^{*}}}
$$

First note that evidently

$$
S_{N}(\Omega) \geq S_{N}\left(\mathbb{R}^{n}\right)=: S_{N},
$$

since every function in $H_{0}^{1}(\Omega)$ may be extended by 0 to an $H^{1}$-function on $\mathbb{R}^{n}$. On the other hand, taking

$$
\widetilde{u}_{\lambda}(x)=\eta(x) u_{\lambda}(x),
$$


where $\eta \in C_{0}^{\infty}(\Omega)$ is a cut-off function, i.e. $\eta \equiv 1$ on a neighborhood $B_{\rho}(0) \subset \Omega$. One then estimates that for $\lambda \rightarrow 0^{+}$

$$
\begin{aligned}
& \int_{\Omega}\left|\nabla \widetilde{u}_{\lambda}\right|^{2} d x=\int_{\Omega}\left|\nabla u_{\lambda}\right|^{2}+O\left(\lambda^{N-2}\right)=S_{N}^{N / 2}+O\left(\lambda^{N-2}\right) \\
& \int_{\Omega}\left|\widetilde{u}_{\lambda}\right|^{2^{*}} d x=\int_{\Omega}\left|u_{\lambda}\right|^{2^{*}} d x+O\left(\lambda^{N}\right)=S_{N}^{N / 2}+O\left(\lambda^{N}\right)
\end{aligned}
$$

and thus

$$
S_{N}(\Omega) \leq \frac{\int_{\Omega}\left|\nabla \widetilde{u}_{\lambda}\right|^{2} d x}{\left(\int_{\Omega}\left|\widetilde{u}_{\lambda}\right|^{2^{*}} d x\right)^{\frac{2}{2^{*}}}}=\frac{S_{N}^{N / 2}}{S_{N}^{N / N^{*}}}+O\left(\lambda^{N-2}\right)=S_{N}+O\left(\lambda^{N-2}\right) .
$$

From this we conclude that for any domain $\Omega \subset \mathbb{R}^{N}$

$$
S_{N}(\Omega)=S_{N}
$$

and that $\left(\widetilde{u}_{\lambda}\right)$ is a minimizing sequence for $S_{N}$ in $H_{0}^{1}(\Omega)$ which is non-compact in $L^{2^{*}}(\Omega)$.

As already mentioned, the above stated results on the equations with subcritical and critical growth seem to imply that we have a complete result: that is

- compactness and existence of nontrivial solutions in the subcritical case in bounded domains

- loss of compactness due to the appearance of a group invariance, and loss of solutions (in starshaped domains) in the critical case.

Indeed, as pointed out by $\mathrm{H}$. Brezis, this seemingly complete result blocked further research for many years - until H. Brezis and L. Nirenberg published their groundbreaking result, see [10]:

\section{The Brezis-Nirenberg result:}

As mentioned above, the equation with "pure critical growth" (2.2) has no nontrivial solution if $\Omega$ is bounded and starshaped. From the variational point of view, this is due to the lack of compactness, caused by the concentration phenomenon. The crucial observation by H. Brezis and L. Nirenberg is that this concentration is the only way in which compactness can be lost. And if compactness is lost due to concentration, then this happens at precise energy levels (the energy of the concentrating instantons). Brezis and Nirenberg consider in [10] an equation with critical growth and with a lower order perturbation, and then search for solutions by minimizing a suitable constrained energy functional. They then calculate the lowest "level of non-compactness", i.e. the limit value of the functional along the concentrating instantons. Finally, they show that the actual minimum value of the functional is below this "value of non-compacntess", and conclude that hence the minimum is attained. 
More precisely, they consider, for $0<\lambda<\lambda_{1}$, the equation

$$
\left\{\begin{aligned}
-\Delta u & =\lambda u+u^{\frac{n+2}{n-2}}, & & x \in \Omega \\
u & =0 & & x \in \partial \Omega \\
u & >0 & & \text { on } \Omega,
\end{aligned}\right.
$$

and look for solutions by considering the minimzation problem

$$
m:=\inf _{\left\{u \in H_{0}^{1}(\Omega) \backslash\{0\}\right.} \frac{\int_{\Omega}\left(|\nabla u|^{2}-\lambda|u|^{2}\right) d x}{\left(\int_{\Omega}|u|^{2^{*}} d x\right)^{2 / 2^{*}}} .
$$

The only non-compactness level for this minimization is again the level $S_{N}$, since along the concentrating instantons $\int_{\Omega}\left|\widetilde{u}_{\lambda}\right|^{2} \rightarrow 0$, for $\lambda \rightarrow 0$. We now show that $m<S_{N}$. For this we do a more precise estimate: indeed, one can estimate explicitely that (for $N \geq 5$ ):

$$
\int_{\Omega}\left|\widetilde{u}_{\lambda}\right|^{2} d x \geq c \lambda^{2}, \text { for some } c>0, \text { and } \lambda>0 \text { small . }
$$

Thus we get, using the sequence $\left(\widetilde{u}_{\lambda}\right)$ and the estimates $(2.8)$

$$
m \leq S_{N}+O\left(\lambda^{N-2}\right)-c \lambda^{2}<S_{N},(N \geq 5),
$$

for $\lambda>0$ sufficiently small. Thus we have confirmed that the infimum $m$ lies below the non-compactness level $S_{N}$, and hence it is attained! (Similar estimates hold for $N=4$, and with some restricitons for $N=3$ ).

This result is by now classical, and has had an enormous influence on the research of the last 25 years.

\section{The case of dimension $N=2$}

The case of dimension $N=2$ is special, since the corresponding Sobolev space $H_{0}^{1}(\Omega), \Omega \subset \mathbb{R}^{2}$, is a borderline case for Sobolev embeddings: one has

$$
W_{0}^{1,2}(\Omega) \subset L^{p}(\Omega), \text { for all } p \geq 1,
$$

but

$$
W_{0}^{1,2}(\Omega) \nsubseteq L^{\infty}(\Omega)
$$

indeed, the function $\log \log (x))$ belongs to $W^{1,2}\left(B_{1}(0)\right)$, but not to $L^{\infty}(\Omega)$.

So, one is lead to ask if there is another kind of maximal growth in this situation. And indeed, this is the result of Pohozaev [41] and Trudinger [49], 
and which is now called the Trudinger inequality: it says that if $\Omega \subset \mathbb{R}^{2}$ is a bounded domain, then

$$
u \in W_{0}^{1,2}(\Omega) \quad \Longrightarrow \quad \int_{\Omega} e^{|u|^{2}} d x \leq c
$$

We can express this fact alternatively in terms of an embedding. For this we need to introduce the notion of Orlicz space which generalize the $L^{p}$-spaces. Let $\varphi(t)=e^{t^{2}}-1$. This is a so-called $N$-function (see section 5 below, where Orlicz spaces will be discussed in more detail). Let $K_{M}=\left\{u: \Omega \rightarrow \mathbb{R}, \int_{\Omega} \varphi(u)<\right.$ $+\infty\}$. The Orlicz space $L_{\varphi}$ is the linear vector space generated by $K_{M}$. For more details we refer to section 5. The result of Pohozaev and Trudinger now says that one has a continuous embedding

$$
H_{0}^{1}\left(\Omega \subset L_{\varphi}(\Omega), \text { for } \Omega \subset \mathbb{R}^{2}\right. \text { bounded }
$$

and

$$
H_{0}^{1}(\Omega) \subset L_{\psi}(\Omega) \text { compact, for any } \psi \prec \prec \varphi,
$$

where $\psi \prec \prec \varphi$ means that $\psi$ increases essentially more slowly than $\varphi$, see section 5 .

Inequality (3.1) was improved and made precise by J. Moser [38] who proved that:

$$
\sup _{\|\nabla u\|_{L^{2}} \leq 1} \int_{\Omega} e^{\alpha u^{2}} d x \quad \begin{cases}\leq c, & \text { if } \alpha \leq 4 \pi \\ =+\infty, & \text { if } \alpha>4 \pi\end{cases}
$$

One can now ask if the "critical growth" (3.1) produces similar phenomena for equation (2.1) as the case $N \geq 3$; indeed, one has many similarities, but also remarkable differences.

Loss of compactness: Similarly as in the case $N \geq 3$, we can give an explicit sequence $\left(u_{n}\right)$ which is bounded in $H_{0}^{1}(\Omega)$, and such that $\left(u_{n}\right)$ has no convergent subsequence in $L_{\varphi}$. For simplicity, assume that $\Omega=B_{1}(0)$, the unit ball. Let

$$
w_{n}=\frac{1}{\sqrt{2 \pi}}\left\{\begin{array}{cc}
(\log n)^{1 / 2} & \text { if } 0 \leq|x| \leq \frac{1}{n} \\
\frac{\log \frac{1}{|x|}}{(\log n)^{1 / 2}} & \text { if } \frac{1}{n} \leq|x| \leq 1
\end{array}\right.
$$

One checks easily that $\int_{B_{1}}\left|\nabla w_{n}\right|^{2} d x=1$, and hence $w_{n} \rightarrow w$. Furthermore, one checks that $\int_{B_{1}}\left(e^{w_{n}^{2}}-1\right) d x \rightarrow \pi$. On the other hand, we have pointwise $w_{n}(x) \rightarrow 0$, for any $x \neq 0$, and hence $w=0$. From this one concludes that there cannot exist a subsequence with $\left\|w_{n_{k}}-w\right\|_{L_{\varphi}} \rightarrow 0$.

Group invariance: A fundamental difference to the case $N \geq 3$ is that no analogue of the group invariance in $N \geq 3$ is known for the case $N=2$. 
Connected with this, also no identity of Pohozaev type is known for dimension $N=2$, which could be important for obtaining non-existence results.

Before discussing further the issue of existence and non-existence, let us give a more precise notion of critical growth: We say that $f \in C(\mathbb{R})$ has subcritical growth if $f(t) \prec \prec e^{t^{2}}-1$ (see (3.2) and section 5), i.e. if

$$
\lim _{|t| \rightarrow \infty} \frac{f(t)}{e^{\alpha t^{2}}}=0, \text { for every } \alpha>0
$$

and $f(t)$ has critical growth if there exists $\alpha_{0}>0$ such that

$$
\lim _{|t| \rightarrow \infty} \frac{f(t)}{e^{\alpha t^{2}}}=0 \quad \text { if } \alpha>\alpha_{0} \quad, \quad \text { and } \quad \lim _{|t| \rightarrow \infty} \frac{f(t)}{e^{\alpha t^{2}}}=+\infty \quad \text { if } 0<\alpha<\alpha_{0} .
$$

We first consider

\subsection{Subcritical growth}

Concerning subcritical growth, one has the following existence result for equation (2.1):

Theorem 3.1 (see [3], [17])

Assume that $f \in C(\mathbb{R})$ satisfies

(H1) there exist constants $t_{0}>0$ and $M>0$ such that

$$
\begin{aligned}
& 0<F(t)=\int_{0}^{t} f(s) d s \leq M|f(t)|, \forall|t| \geq t_{0} \\
& 0<F(t) \leq \frac{1}{2} f(t) t, \forall t \in \mathbb{R} \backslash\{0\}
\end{aligned}
$$

Then equation (2.1), with $\Omega \subset \mathbb{R}^{2}$ a bounded domain, has a nontrivial solution. Moreover, if $f(t)$ is an odd function in $t$, then equation (2.1) has infinitely many solutions.

Proof. The proof follows (by now) standard lines: the assumptions guarantee that the functional has a mountain pass structure around the origin, cf. [5, 43]. The subcritical growth yields compactness, cf. (3.2), and hence the critical level is attained.

\subsection{Critical growth}

We consider now equation (2.1) with critical growth in the sense specified in (3.5) above. For the case $N=2$ the situation is more complicated than for dimensions $N \geq 3$, and the known results are less complete. The difficulties start already with the fact that there is no natural "model problem" for the 
critical case. Thus, let us write the "critical" equation (with $\alpha_{0}=4 \pi$, see (3.5)) in the form

$$
-\Delta u=h(u) e^{4 \pi u^{2}}=e^{4 \pi u^{2}+\log h(u)}, \Omega ; u=0, \partial \Omega
$$

where $h \in C(\mathbb{R})$ is subcritical, i.e. satisfying condition (3.4). Thus, $\log h(u)$ is a "lower order perturbation" of the principal growth term $4 \pi u^{2}$, and in analogy to the Brezis-Nirenberg case we can ask for conditions on $h(t)$ such that we have again the situation of non-existence or existence of solutions.

Related to the study of this question is the behavior of the supremum in (3.3). Indeed, it came as a surprise when L. Carleson - A. Chang [12] proved in 1986 that the supremum in (3.3) is attained on the unit ball in $\mathbb{R}^{2}$. In fact, consider for comparison the following maximization problem, for $N \geq 3$

$$
\sup _{\|\nabla u\|_{L^{2} \leq 1}} \int_{\Omega}|u|^{2^{*}} d x=M
$$

This problem corresponds to the minimzation problem (2.6), and by (2.9) it is clear that

$$
M=\frac{1}{S_{N}^{\frac{N}{N-2}}} .
$$

Furthermore, by the remarks made following (2.6), the supremum in (3.6) is never attained if $\Omega \neq \mathbb{R}^{N}$.

Thus, the result of Carleson and Chang (for $\Omega=B_{1}(0)$ ) is in striking contrast to the case $N \geq 3$. We remark that the result of Carleson and Chang was extended to arbitrary domains in $\mathbb{R}^{2}$ by M. Flucher [24]).

Carleson and Chang prove their result by the following steps: Let $\left\{u_{n}\right\}$ be a maximizing sequence.

- by radial symmetrization, one see that $\left\{u_{n}\right\}$ may be assumed radial, and thus characterized by an ODE (the radial equation)

- if the supremum is not attained, then the maximizing sequence is a "normalized concentrating sequence", i.e. it tends weakly to 0 and concentrates in the origin

- determine an explicit upper bound for any such normalized concentrating sequence $\left(u_{n}\right) \in H_{0}^{1}\left(B_{1}\right)$, namely

$$
\int_{B_{1}(0)} e^{4 \pi u_{n}^{2}} \leq(1+e) \pi
$$

- provide an explicit normalized function $w \in H_{0}^{1}\left(B_{1}\right)$ with

$$
\int_{B_{1}} e^{4 \pi w^{2}}>(1+e) \pi
$$


Clearly, $(1+e) \pi$ takes the rôle of the (highest) non-compactness level, analogous to the situation in $\mathbb{R} \geq 3$ described above, and since the supremum lies above this non-compactness level, it is attained.

In a recent paper by De Figueiredo, do Ó and the author [18] the following explicit normalized concentrating and maximizing sequence for $(1+e) \pi$ was constructed:

For $n \in \mathbb{N}$ set $\delta_{n}=\frac{2 \log n}{n}$ and $A_{n}=\frac{1}{e n^{2}}+O\left(\frac{1}{n^{4}}\right) ;$ then define

$$
y_{n}(|x|)= \begin{cases}\left(\frac{1-\delta_{n}}{n}\right)^{1 / 2} \log \frac{1}{|x|} & , \quad 1 / n \leq|x| \leq 1 \\ \frac{1}{\left(n\left(1-\delta_{n}\right)\right)^{1 / 2}} \log \frac{A_{n}+1}{A_{n}+n|x|}+\left(n\left(1-\delta_{n}\right)\right)^{1 / 2}, & 0 \leq|x| \leq 1 / n\end{cases}
$$

The constants $A_{n}$ are chosen such that $\int_{B_{1}}\left|\nabla u_{n}\right|^{2} d x=1$. This sequence allows to give a new proof of the last step in the argument of Carleson-Chang (and also a generalization of their result): one shows that this sequence approaches the value $(1+e) \pi$ from above, i.e.

$$
\int_{B_{1}} e^{4 \pi u_{n}^{2}} d x>(1+e) \pi, \text { for } n \text { large . }
$$

This is in complete analogy to the case of Brezis-Nirenberg, whereby the sequence (3.7) takes the rôle of the sequence $\widetilde{u}_{\lambda}$, see $(2.7)$.

Problem: In view of this and the above remarks, it is of interest to consider

$$
\sup _{u \in H_{0}^{1}(\Omega),\|u\|=1} \int_{\Omega} h(u) e^{4 \pi u^{2}}=S
$$

and give optimal (= sharp) conditions on the subcritical function $h(t)$ such that the supremum $S$ is attained, respectively not attained.

\subsection{Critical growth: existence}

For the corresponding equation

$$
\left\{\begin{aligned}
-\Delta u & =h(u) e^{4 \pi u^{2}}, \text { in } \Omega \\
u & =0, \text { on } \partial \Omega
\end{aligned}\right.
$$

some progress has been made recently concerning the determination of an optimal subcritical function $h(t)$. We remark that concerning non-existence results, a fundamental difference to the case $N \geq 3$ is that (up to now) no suitable identity of Pohozaev type is known for the case $N=2$.

In [17] the following theorem was proved by de Figueiredo, Miyagaki and the author (see also Adimurthi [2]): 
Theorem 3.2 Assume that $h \in C(\mathbb{R})$ and let $f(s)=h(s) e^{4 \pi s^{2}}$. Assume that H1) $f(0)=0$

H2) there exist constants $s_{0}>0$ and $M>0$ such that

$$
0<F(s)=\int_{0}^{s} f(r) d r \leq M|f(s)|, \forall|s| \geq s_{0}
$$

H3) $0<F(s) \leq \frac{1}{2} f(s) s, \forall s \in \mathbb{R} \backslash\{0\}$

Furthermore, let $d$ denote the inner radius of $\Omega$, i.e.

$$
d:=\text { radius of the largest ball } \subset \Omega
$$

Then equation (3.8) has a nontrivial solution provided that

$$
\lim _{|s| \rightarrow \infty} h(s) s=\beta>\frac{1}{2 \pi d^{2}}
$$

The proof of this theorem follows closely the scheme by Brezis-Nirenberg mentioned above, that is

- determine (explicitly) the level of non compactness

- use an explicit concentrating sequence and the hypothesis on $h(t)$ to show that the min-max level is below this non-compactness level

- thus, compactness is recovered and the existence of a solution follows

The concentrating sequence used in the proof of this theorem is the so-called Moser sequence given by

$$
w_{n}=\frac{1}{\sqrt{2 \pi}} \begin{cases}\frac{1}{(\log n)^{1 / 2}} \log \frac{1}{|x|} & \frac{1}{n} \leq|x| \leq 1 \\ (\log n)^{1 / 2} & 0 \leq|x| \leq \frac{1}{n}\end{cases}
$$

We remark that this sequence is not an optimal concentrating sequence; in fact, one easily calculates that

$$
\lim _{n \rightarrow \infty} \int_{B_{1}} e^{4 \pi w_{n}^{2}}=2 \pi<(1+e) \pi
$$

We remark that the condition $H 4$ ) in Theorem 3.2 may be slightly improved to

$$
\beta>\frac{1}{e \pi d^{2}}
$$

by using the optimal maximizing sequence (3.7) mentioned above instead of the Moser sequence. 


\subsection{Critical growth: non-existence}

Concerning non-existence, only a partial result is known; in the following theorem, the non-existence of a positive radial solution on $\Omega=B_{1}(0)$ is proved under conditions comparable to those of Theorem 3.2.

Theorem 3.3 (de Figueiredo-Ruf [21])

Let $\Omega=B_{1}(0)$. Suppose that $h \in C^{2}(\mathbb{R})$, and that there exist constants $r_{1}>0$ and $\sigma>0$ such that for some constants $K>0, c>0$ :

1. $h(r)=\frac{K}{r} \quad$ for $r \geq r_{1}$

2. $0 \leq h(r) \leq c K r^{1+\sigma}$, for $0 \leq r \leq r_{1}$.

Then there exists a constant $K_{0}>0$ such that for $K<K_{0}$ the equation

$$
\left\{\begin{aligned}
-\Delta u & =h(u) e^{4 \pi u^{2}}, & & \text { in } B_{1}(0) \subset \mathbb{R}^{2} \\
u & =0, & & \text { on } \partial B_{1}(0)
\end{aligned}\right.
$$

has no non-trivial radial solution.

We remark that by Gidas-Ni-Nirenberg [25] any positive solution of equation (3.9) is radial, and hence Theorem 3.3 says that equation (3.9) has no positive solution.

Comparing Theorems 3.2 and 3.3 one notes that Theorem 3.3 assures (under some "technical" conditions) the existence of a positive solution for $\Omega=B_{1}(0)$ provided that asymptotically

$$
h(s) \sim \frac{\beta}{s}, \text { with } \beta>\frac{1}{2 \pi},
$$

while Theorem 3.3 gives non-existence for

$$
h(s)=\frac{\beta}{s} \text { for } s \text { large, and } \beta>0 \text { sufficiently small. }
$$

The proof of Theorem 3.3 uses techniques of the theory of ordinary differential equations, in particular the shooting method. More precisely, considering only the radial solutions on $\Omega=B_{1}(0)$, one can reduce equation (3.9) to the radial equation

$$
\left\{\begin{array}{l}
u_{r} r+\frac{1}{r} u_{r}+h(u) e^{4 \pi u^{2}}=0 \quad \text { in }(0,1) \\
u^{\prime}(0)=u(1)=0
\end{array}\right.
$$

Using the transformation $t=-2 \log \frac{r}{2}$ and setting $y(t)=u(r)$ we obtain

$$
\left\{\begin{array}{l}
-y^{\prime \prime}=h(y) e^{4 \pi y^{2}-t}, \text { for } t>2 \log 2 \\
y(2 \log 2)=0, y^{\prime}(+\infty)=0
\end{array}\right.
$$


That is, we have transformed equation (3.10), which has a singularity in 0 , to equation (3.11) on $(2 \log 2,+\infty)$, thus transporting the singularity in 0 to $+\infty$. The shooting method consists now in considering solutions $y(t)$ of $(3.11)$ with $y^{\prime}(+\infty)=\gamma$, i.e. one shoots horizonally from infinity and tries to land at the point $2 \log 2$. The estimates to achieve this are delicate and lengthy, and are a refinement of the work of Atkinson-Peletier [6].

We summarize: if we assume that the asymptotic condition in the existence theorem 3.2 is optimal (at least on the unit ball $B_{1}$ ), then the major open problem may be stated as follows:

Find a good model equation (i.e. properties of $h(u)$ ) under which one may prove: existence of a non-trivial solution for $\lim _{t \rightarrow \infty} h(t) t>\frac{1}{e \pi}$, and non-existence of a solution for $\lim _{t \rightarrow \infty} h(t) t<\frac{1}{e \pi}$.

As already mentioned, what seems to be missing is a kind of Pohozaev identity to obtain a sharp non-existence result.

\section{$4 \quad$ Elliptic systems, $N \geq 3$}

\subsection{Strongly indefinite functionals}

In this section we begin the discussion of elliptic systems of the following simple form

$$
\left\{\begin{aligned}
-\Delta u=g(v) & \\
-\Delta v=f(u) & \text { in } \Omega \subset \mathbb{R}^{N}, N \geq 3 \\
u=v=0 & \text { on } \partial \Omega
\end{aligned}\right.
$$

where $f, g: \mathbb{R} \rightarrow \mathbb{R}$ are continuous and superlinear functions, i.e.

$$
\frac{f(s)}{s} \rightarrow+\infty \quad, \quad \frac{g(s)}{s} \rightarrow+\infty, \text { as }|s| \rightarrow \infty
$$

These systems are of so-called Hamiltonian form; indeed, we can define the Hamiltonian $H(u, v)=F(u)+G(v)$, where $F$ and $G$ are the primitives of $f$ and $g$ respectively. Then we get the system: $-\Delta u=H_{v}(u, v)$ and $-\Delta v=H_{u}(u, v)$.

As in the scalar case, our first interest is to find the maximal or "critical" growth for the nonlinearities $f$ and $g$.

We can employ the same procedure as for the scalar equation: Write down a functional for the system (such that critical points yield weak solutions), and then find the appropriate function space on which the functional is well defined. The functional we choose is the following:

$$
I(u, v)=\int_{\Omega} \nabla u \nabla v d x-\int_{\Omega} F(u) d x-\int_{\Omega} G(v) d x
$$


As a first attempt, we may define the functional on the space

$$
E:=H_{0}^{1}(\Omega) \times H_{0}^{1}(\Omega)
$$

by estimating

$$
\left|\int_{\Omega} \nabla u \nabla v d x\right| \leq\|\nabla u\|_{L^{2}}\|\nabla v\|_{L^{2}}=\|u\|_{H_{0}^{1}}\|v\|_{H_{0}^{1}}
$$

In order to have the functional well defined and continuous, we then obtain as in the scalar case the following growth conditions for the primitives $F(s)=$ $\int_{0}^{s} g(t) d t$ and $G(s)=\int_{0}^{s} g(t) d t$ :

$$
F(s) \leq c_{1}+d_{1}|s|^{2^{*}}, \quad G(s) \leq c_{2}+d_{2}|s|^{2^{*}}
$$

Suppose now that $(\bar{u}, \bar{v}) \in E$ is a critical point of $I(u, v)$; then we have

$$
\int_{\Omega} \nabla \varphi \nabla \bar{v}+\int_{\Omega} \nabla \bar{u} \nabla \psi=\int_{\Omega} f(\bar{u}) \varphi+\int_{\Omega} g(\bar{v}) \psi, \forall(\varphi, \psi) \in E .
$$

Choosing in particular the directions $(\varphi, 0)$ and $(0, \psi)$, we obtain that $(\bar{u}, \bar{v})$ is a weak solution of system (4.1).

Note that the functional $I(u, v)$ has a more complicated structure than the functionals considered up to now: the quadratic term $\int_{\Omega} \nabla u \nabla v d x$ is strongly indefinite near the origin; indeed, it is positively resp. negatively definite on infinite dimensional subspaces of $E$. In recent years much research has been devoted to the study of such situations, we refer to [35], [33] and [8]. We will describe below, in a more general situation, a detailed approach for such problems.

\subsection{The critical hyperbola}

We have seen above that the "natural choice" of space $E=H_{0}^{1}(\Omega) \times H_{0}^{1}(\Omega)$ leads to the known Sobolev growth restriction for both nonlinaerities $F(s)$ and $G(s)$.

However, by a different choice of the space $E$, this result can be considerably generalized. In independent works by Hulshof - van der Vorst [28] and de Figueiredo - Felmer [15] it was shown that one may have different maximal growths for $F$ and $G$; more precisely, the condition for the two nonlinearities is given by

$$
F(s) \leq c_{1}+d_{1}|s|^{p+1}, \quad G(s) \leq c_{2}+d_{2}|s|^{q+1}
$$

with $p+1$ and $q+1$ satisfying the condition

$$
\frac{1}{p+1}+\frac{1}{q+1}=1-\frac{2}{N}
$$


This is the so-called critical hyperbola. We will show in the next sections in some detail how the critical hyperbola arises. Indeed, we will see that it can be obtained in two quite different ways, first in a Hilbert space setting, working with fractional Sobolev spaces $H^{s}$, and then in a Banach space setting, working with $W^{1, \alpha}-$ spaces.

It is interesting that this critical hyperbola has many of the features of the critical exponents, namely there is

- compactness below the critical hyperbola, i.e. for nonlinearities with exponential growths $p$ and $q$ with

$$
\frac{1}{p+1}+\frac{1}{q+1}>1-\frac{2}{N}
$$

and in consequence, existence of solutions for such equations; see subsection 5.4 below.

- loss of compactness and concentration phenomena for systems with critical growth, i.e. when the exponents lie on the critical hyperbola, see subsection 8.2

- non-existence of solutions for the pure power case and on starshaped domains, due to a Pohozaev (or Rellich) type inequality; see subsection 8.1

- existence of instantons; however, in contrast to the scalar equation, these are not explicitly known, but it is possible to derive their asymptotic behavior, see subsection 8.3

- group invariance

\subsection{The $H^{s}$-approach}

In this section we will use fractional Sobolev spaces $H^{s}$ on which the functional $I(u, v)$ will be defined.

\subsubsection{Fractional Sobolev spaces and the functional setting}

To describe the idea of de Figueiredo - Felmer [15] and Hulshoff - van der Vorst [28], we begin by defining fractional Sobolev spaces.

Consider the Laplacian as the operator

$$
-\Delta: H^{2}(\Omega) \cap H_{0}^{1}(\Omega) \subset L^{2}(\Omega) \rightarrow L^{2}(\Omega),
$$

and $\left\{e_{i}\right\}_{i=1}^{\infty}$ a corresponding system of orthogonal and $L^{2}$-normalized eigenfunctions, with eigenvalues $\left\{\lambda_{i}\right\}$. Then, writing

$$
u=\sum_{n=1}^{\infty} a_{n} e_{n}, \text { with } a_{n}=\int_{\Omega} u e_{n} d x
$$


we set

$$
E^{s}=\left\{u \in L^{2}(\Omega): \sum_{n=1}^{\infty} \lambda_{n}^{s}\left|a_{n}\right|^{2}<\infty\right\}
$$

and

$$
A^{s} u=\sum_{n=1}^{\infty} \lambda_{n}^{s / 2} a_{n} e_{n}, \forall u \in D\left(A^{s}\right):=E^{s} .
$$

The spaces $E^{s}$ are fractional Sobolev spaces with the inner product

$$
(u, v)_{s}=\int_{\Omega} A^{s} u A^{s} v d x
$$

see Lions-Magenes [37].

In the next Lemma we collect a few properties of the operators $A^{s}$ and the spaces $E^{s}$.

Lemma 4.1 Let $s>0$ and $t>0$.

1) $z \in E^{s}$ iff $A^{s} z \in L^{2}$, and $\|z\|_{E^{s}}=\left\|A^{s} z\right\|_{L^{2}}$

2) Let $z \in E^{s+t}=E^{2}=H^{2}$; then $A^{s+t} z=A^{s} A^{t} z=A^{t} A^{s} z$.

Proof. 1) follows immediately from the definitions.

2) we have

$$
A^{s+t} z=\sum_{i \in \mathbb{N}} \alpha_{i} \lambda_{i}^{(s+t) / 2} e_{i}=\sum_{i \in \mathbb{N}} \alpha_{i} \lambda_{i}^{s / 2} \lambda_{i}^{t / 2} e_{i}=A^{s} \sum_{i \in \mathbb{N}} \alpha_{i} \lambda_{i}^{t / 2} e_{i}=A^{s} A^{t} z
$$

With these definitions, we now define the functional

$$
\begin{aligned}
& I: E^{s} \times E^{t} \rightarrow \mathbb{R} \\
& I(u, v)=\int_{\Omega} A^{s} u A^{t} v-\int_{\Omega}(F(u)+G(v)) d x
\end{aligned}
$$

with $s$ and $t$ such that $s+t=2$; loosely speaking, this means that we distribute the two derivatives given in the first term of the functional $I$ differently on the variables $u$ and $v$.

The first term of $I(u, v)$ is well defined on the space $E^{s} \times E^{t}$ by the estimate

$$
\left|\int_{\Omega} A^{s} u A^{t} v d x\right| \leq\left\|A^{s} u\right\|_{L^{2}}\left\|A^{t} v\right\|_{L^{2}}=\|u\|_{E^{s}}\|v\|_{E^{t}}
$$

By the Sobolev embedding theorem we have continuous embeddings

$$
E^{s} \subset L^{p+1}(\Omega), \text { if } \frac{1}{p+1} \geq \frac{1}{2}-\frac{s}{N}
$$


and these embeddings are compact if $\frac{1}{p+1}>\frac{1}{2}-\frac{s}{N}$, and similarly for the embedding

$$
E^{t} \subset L^{q+1}(\Omega), \text { if } \frac{1}{q+1} \geq \frac{1}{2}-\frac{s}{N} .
$$

Summing the two conditions above we now obtain the growth restrictions

$$
\frac{1}{p+1}+\frac{1}{q+1} \geq 1-\frac{s+t}{N}=1-\frac{2}{N}
$$

i.e. we have found the critical hypberbola.

Of course, it is crucial to recuperate from critical points $(u, v)$ of this functional (weak) solutions of system (4.1). We state this in the following

Proposition 4.2 Suppose that $(u, v) \in E^{s} \times E^{t}$ is a critical point of the functional $I$, i.e. $u$ and $v$ are weak solutions of the system

$$
\left\{\begin{aligned}
\int_{\Omega} A^{s} u A^{t} \phi & =\int_{\Omega} v^{p} \phi, \forall \phi \in E^{s} \\
\int_{\Omega} A^{s} \psi A^{t} v & =\int_{\Omega} f(u) \psi, \forall \psi \in E^{t} .
\end{aligned}\right.
$$

Then $v \in W^{2, \frac{p+1}{p}}(\Omega) \cap W_{0}^{1, \frac{p+1}{p}}(\Omega)$ and $u \in W^{2, q}(\Omega) \cap W_{0}^{1, q}(\Omega), \forall q \geq 1$, and hence $u$ and $v$ are "strong" solutions of (4.4), i.e.

$$
\left\{\begin{aligned}
\int_{\Omega}(-\Delta u) \phi & =\int_{\Omega} g(v) \phi, \forall \phi \in C_{0}^{\infty}(\Omega) \\
\int_{\Omega}(-\Delta v) \psi & =\int_{\Omega} f(u) \psi, \forall \psi \in C_{0}^{\infty}(\Omega) .
\end{aligned}\right.
$$

From this proposition follows by standard bootstrap arguments that $u$ and $v$ are classical solutions of (4.1) if $f$ and $\Omega$ are smooth.

For the proof of the proposition, see de Figueiredo - Felmer [15].

\subsubsection{Compactness and existence of solutions for systems with subcritical growth}

Subcritical growth is given for nonlinearities whose growth restrictions satisfy

$$
\frac{1}{p+1}+\frac{1}{q+1}>1-\frac{1}{N}
$$

Remark 4.3 To simplify the exposition, we state the theorems and give the proof for the so-called "model problem", i.e. system (4.1) with polynomial-type nonlinearities, i.e. $f(u)=u^{p}$ and $g(v)=v^{q}$. For the more general versions, the reader is referred to the literature. 
Thus, we consider the system

$$
\left\{\begin{aligned}
-\Delta u=v^{q} & \text { in } \Omega \subset \mathbb{R}^{N}, N \geq 3 \\
-\Delta v=u^{p} & \text { on } \partial \Omega \\
u=v=0 &
\end{aligned}\right.
$$

We then have

Theorem 4.4 For subcritical growth, i.e. if $p+1$ and $q+1$ satisfy (4.6), system (4.7) has a nontrivial solution.

Proof. We have defined the functional

$$
\begin{aligned}
& I: E^{s}(\Omega) \times E^{t}(\Omega) \rightarrow \mathbb{R} \\
& I(u, v)=\int_{\Omega} A^{s} u A^{t} v d x-\frac{1}{p+1} \int_{\Omega}|u|^{p+1} d x-\frac{1}{q+1} \int_{\Omega}|v|^{q+1} d x
\end{aligned}
$$

An inherent difficulty to systems of type (4.1) is that the associated functional is strongly indefinite, in the sense that near the origin it is positive resp. negative definite on infinite dimensional subspaces. This precludes a direct application of the (by now classical) "linking theorems" of Critical Point Theory. In recent years, several approaches have been devised to overcome this problem:

- approximation by finite dimensional problems

- introduction of a new (weak-strong) topology: W. Kryszewski and A. Szulkin [33], Th. Bartsch and Y. Ding [8]

- infinite linking with compactness: S. Li- M. Willem [35]

We will use the last method to prove the above theorem. In later chapters, we will also show an application of the first method.

In [35], S. Li and M. Willem prove the following theorem:

Theorem 4.5 Let $\Phi: E \rightarrow \mathbb{R}$ be a strongly indefinite $C^{1}$-functional satisfying

A1) $\Phi$ has a local linking at the origin, i.e. for some $r>0$ :

$$
\Phi(z) \geq 0 \text { for } z \in E^{+},\|z\|_{E} \leq r \quad, \quad \Phi(z) \leq 0, \text { for } z \in E^{-},\|z\|_{E} \leq r .
$$

A2) $\Phi$ maps bounded sets into bounded sets.

A3) Let $E_{n}^{+}$be any $n$-dimensional subspace of $E^{+}$; then $\phi(z) \rightarrow-\infty$ as $\|z\| \rightarrow \infty, z \in E_{n}^{+} \oplus E^{-}$.

A4) $\Phi$ satisfies the Palais-Smale condition (PS) (Li-Willem [35] require a weaker "(PS*)-condition", however, in our case the classical (PS) condition will be satisfied). 
Then $\Phi$ has a nontrivial critical point.

We now verify that our functional $I(u, v)$ satisfies the assumptions of this theorem. We assume, without restricting generality, that

$$
1<q \leq p, \text { and hence } s \geq t
$$

Also, we may assume that e.g. the embedding $E^{t} \subset L^{q+1}$ is compact.

First, it is clear, with the choices of $s$ and $t$ made above, that $I(u, v)$ is a $C^{1}$-functional on $E^{s} \times E^{t}$.

A1) Following de Figueiredo-Felmer [15] we can define the spaces

$E^{+}=\left\{\left(y, A^{s-t} y\right) \mid y \in E^{s}\right\} \subset E^{s} \times E^{t}, E^{-}=\left\{\left(y,-A^{s-t} y\right) \mid y \in E^{s}\right\} \subset E^{s} \times E^{t}$,

which give a natural splitting $E^{+} \oplus E^{-}=E$. It is easy to see that $I(u, v)$ has a local linking with respect to $E^{+}$and $E^{-}$at the origin.

A2) Let $B \subset E^{s} \times E^{t}$ be a bounded set, i.e. $\|u\|_{E^{s}} \leq c,\|v\|_{E^{t}} \leq c$, for all $(u, v) \in B$. Then, by the embeddings $E^{s} \subset L^{p+1}$ and $E^{t} \subset L^{q+1}$

$$
\begin{aligned}
|I(u, v)| & \leq\left\|A^{s} u\right\|_{L^{2}}\left\|A^{t} v\right\|_{L^{2}}+\frac{1}{p+1} \int_{\Omega}|u|^{p+1}+\frac{1}{q+1} \int_{\Omega}|v|^{q+1} \\
& \leq\|u\|_{E^{s}}\|v\|_{E^{t}}+c\|u\|_{E^{s}}^{p+1}+c\|v\|_{E^{t}}^{q+1} \leq C
\end{aligned}
$$

A3) Let $z_{k}=z_{k}^{+}+z_{k}^{-} \in E=E_{n}^{+} \oplus E^{-}$denote a sequence with $\left\|z_{k}\right\| \rightarrow \infty$. By the above, $z_{k}$ may be written as

$$
z_{k}=\left(u_{k}, A^{s-t} u_{k}\right)+\left(v_{k},-A^{s-t} v_{k}\right) \text {, with } u_{k} \in E_{n}^{s}, v_{k} \in E^{s},
$$

where $E_{n}^{s}$ denotes an $n$-dimensional subspace of $E^{s}$. Thus, the functional $I\left(z_{k}\right)$ takes the form

$$
\begin{aligned}
I\left(z_{k}\right)= & \int_{\Omega} A^{s} u_{k} A^{t} A^{s-t} u_{k}-\int_{\Omega} A^{s} v_{k} A^{t} A^{s-t} v_{k}- \\
& \quad-\frac{1}{p+1} \int_{\Omega}\left|u_{k}+v_{k}\right|^{p+1}-\frac{1}{q+1} \int_{\Omega}\left|A^{s-t}\left(u_{k}-v_{k}\right)\right|^{q+1} \\
= & \left.\int_{\Omega}\left|A^{s} u_{k}\right|^{2}-\int_{\Omega}\left|A^{s} v_{k}\right|^{2}-\frac{1}{p+1} \int_{\Omega} \mid u_{k}+v_{k}\right)\left.\right|^{p+1}-\frac{1}{q+1} \int_{\Omega}\left|A^{s-t}\left(u_{k}-v_{k}\right)\right|^{q+1}
\end{aligned}
$$

Note that $\left\|z_{k}\right\| \rightarrow \infty \Longleftrightarrow \int\left|A^{s} u_{k}\right|^{2}+\int\left|A^{t} v_{k}\right|^{2}=\left\|u_{k}\right\|_{E^{s}}^{2}+\left\|v_{k}\right\|_{E^{s}}^{2} \rightarrow \infty$.

Now, if

1) $\left\|u_{k}\right\|_{E^{s}} \leq c$, then $\left\|v_{k}\right\|_{E^{s}} \rightarrow \infty$, and then $J\left(z_{k}\right) \rightarrow-\infty$

2) $\left\|u_{k}\right\|_{E^{s}} \rightarrow \infty$, then we estimate (c, $c_{1}, c_{2}$ and $d$ are positive constants)

$$
\begin{gathered}
\left.\left.\int_{\Omega} \mid u_{k}+v_{k}\right)\left.\right|^{p+1} \geq\left. c\left(\int_{\Omega} \mid u_{k}+v_{k}\right)\right|^{2}\right)^{\frac{p+1}{2}}-c \\
\geq c_{1}\left\|u_{k}+v_{k}\right\|_{L^{2}}^{p+1}-c \geq c_{2}\left\|u_{k}+v_{k}\right\|_{L^{2}}^{q+1}-\bar{c}
\end{gathered}
$$


and, by Poincaré's inequality, since $s \geq t$

$$
\int_{\Omega}\left|A^{s-t}\left(u_{k}-v_{k}\right)\right|^{q+1} \geq c_{1}\left\|A^{s-t}\left(u_{k}-v_{k}\right)\right\|_{L^{2}}^{q+1}-c \geq c_{2}\left\|u_{k}-v_{k}\right\|_{L^{2}}^{q+1}-\bar{c}
$$

and hence we obtain the estimate

$$
I\left(z_{k}\right) \leq\left\|u_{k}\right\|_{E^{s}}^{2}-c\left(\left\|u_{k}+v_{k}\right\|_{L^{2}}^{q+1}+\left\|u_{k}-v_{k}\right\|_{L^{2}}^{q+1}\right)+d
$$

Since $\phi(t)=t^{q+1}$ is convex, we have $\frac{1}{2}(\phi(t)+\phi(s)) \geq \phi\left(\frac{1}{2}(s+t)\right)$, and hence

$$
\begin{aligned}
I\left(z_{k}\right) & \leq \frac{1}{2}\left\|u_{k}\right\|_{E^{s}}^{2}-c \frac{1}{2^{q}}\left(\left\|u_{k}-v_{k}\right\|_{L^{2}}+\left\|u_{k}+v_{k}\right\|_{L^{2}}\right)^{q+1}+d \\
& \leq \frac{1}{2}\left\|u_{k}\right\|_{E^{s}}^{2}-c \frac{1}{2^{q}}\left\|u_{k}\right\|_{L^{2}}^{q+1}+d
\end{aligned}
$$

Since on $E_{n}^{s}$ the norms $\left\|u_{k}\right\|_{E^{s}}$ and $\left\|u_{k}\right\|_{L^{2}}$ are equivalent, we conclude that also in this case $J\left(z_{k}\right) \rightarrow-\infty$.

A4) Let $\left\{z_{n}\right\} \subset E$ denote a (PS)-sequence, i.e. such that

$$
\left|I\left(z_{n}\right)\right| \rightarrow c, \quad \text { and }\left|\left(I^{\prime}\left(z_{n}\right), \eta\right)\right| \leq \epsilon_{n}\|\eta\|_{E}, \forall \eta \in E, \quad \text { and } \epsilon_{n} \rightarrow 0
$$

We first show:

Lemma 4.6 The (PS)-sequence $\left\{z_{n}\right\}$ is bounded in E.

Proof. By (4.8) we have for $z_{n}=\left(u_{n}, v_{n}\right) \in E$

$$
\begin{aligned}
& I\left(u_{n}, v_{n}\right)=\int_{\Omega} A^{s} u_{n} A^{t} v_{n}-\frac{1}{p+1} \int_{\Omega}\left|u_{n}\right|^{p+1}-\frac{1}{q+1} \int_{\Omega}\left|v_{n}\right|^{q+1} \rightarrow c \\
& I^{\prime}\left(u_{n}, v_{n}\right)(\phi, \psi)=\int_{\Omega} A^{s} u_{n} A^{t} \psi+\int_{\Omega} A^{t} v_{n} A^{s} \phi-\int_{\Omega} u_{n}^{p} \psi \\
&-\int_{\Omega} v_{n}^{q} \phi=\epsilon_{n}\|(\phi, \psi)\|_{E}
\end{aligned}
$$

Choosing $(\phi, \psi)=\left(u_{n}, v_{n}\right) \in E^{s} \times E^{t}$ we get by $(4.10)$

$$
2 \int_{\Omega} A^{s} u_{n} A^{t} v_{n}-\int\left|u_{n}\right|^{p+1}-\int_{\Omega}\left|v_{n}\right|^{q+1}=\epsilon_{n}\left(\left\|u_{n}\right\|_{E^{t}}+\left\|v_{n}\right\|_{E^{s}}\right)
$$

and subtracting this from $2 I\left(u_{n}, v_{n}\right)$ we obtain

$$
\left(1-\frac{2}{p+1}\right) \int_{\Omega}\left|u_{n}\right|^{p+1}+\left(1-\frac{2}{q+1}\right) \int_{\Omega}\left|v_{n}\right|^{q+1} \leq C+\epsilon_{n}\left(\left\|u_{n}\right\|_{E^{s}}+\left\|v_{n}\right\|_{E^{t}}\right)
$$

and thus

$$
\int_{\Omega}\left|u_{n}\right|^{p+1} \leq C+\epsilon_{n}\left(\left\|u_{n}\right\|_{E^{s}}+\left\|v_{n}\right\|_{E^{t}}\right)
$$




$$
\int_{\Omega}\left|v_{n}\right|^{q+1} \leq C+\epsilon_{n}\left(\left\|u_{n}\right\|_{E^{s}}+\left\|v_{n}\right\|_{E^{t}}\right)
$$

Next, note that $A^{s-t} u_{n} \in E^{t}$; indeed, $u_{n} \in E^{s}$ implies that $A^{t}\left(A^{s-t} u_{n}\right)=$ $A^{s} u_{n} \in L^{2} \Longleftrightarrow A^{s-t} u_{n} \in E^{t}$.

Thus, choosing $(\phi, \psi)=\left(0, A^{s-t} u_{n}\right) \in E^{s} \times E^{t}$ in (4.10) we get

$$
\int_{\Omega}\left|A^{s} u_{n}\right|^{2}=\int_{\Omega} v_{n}^{q} A^{s-t} u_{n}+\epsilon_{n}\left\|A^{s-t} u_{n}\right\|_{E^{t}}
$$

and hence by Hölder

$$
\left\|u_{n}\right\|_{E^{s}}^{2}=\left\|A^{t} u_{n}\right\|_{L^{2}}^{2} \leq\left(\int_{\Omega}\left|v_{n}\right|^{q+1}\right)^{\frac{q}{q+1}}\left(\int_{\Omega}\left|A^{s-t} u_{n}\right|^{p+1}\right)^{\frac{1}{p+1}}+\epsilon_{n}\left\|u_{n}\right\|_{E^{s}}
$$

Noting that

$$
\left\|A^{s-t} u_{n}\right\|_{q+1} \leq c\left\|A^{s-t} u_{n}\right\|_{E^{s}}=c\left\|A^{s} u_{n}\right\|_{L^{2}}=c\left\|u_{n}\right\|_{E^{s}}
$$

we obtain, using (4.14)

$$
\left\|u_{n}\right\|_{E^{s}}^{2} \leq\left[C+\epsilon_{n}\left(\left\|u_{n}\right\|_{E^{s}}+\left\|v_{n}\right\|_{E^{t}}\right)\right]^{q /(q+1)} \cdot c\left\|u_{n}\right\|_{E^{s}}+\epsilon_{n}\left\|u_{n}\right\|_{E^{s}}
$$

and thus

$$
\left\|u_{n}\right\|_{E^{s}} \leq C+\epsilon_{n}\left(\left\|u_{n}\right\|_{E^{s}}+\left\|v_{n}\right\|_{E^{t}}\right)
$$

Similarly as above we note that $A^{t-s} v_{n} \in E^{s}$, and thus, choosing $(\phi, \psi)=$ $\left(A^{t-s} v_{n}, 0\right) \in E^{s} \times E^{t}$ in (4.10) we obtain as above

$$
\left\|v_{n}\right\|_{E^{t}} \leq C+\epsilon_{n}\left(\left\|v_{n}\right\|_{E^{t}}+\left\|u_{n}\right\|_{E^{s}}\right)
$$

Joining (4.15) and (4.16) we finally get

$$
\left\|u_{n}\right\|_{E^{s}}+\left\|v_{n}\right\|_{E^{t}} \leq C+2 \epsilon_{n}\left(\left\|u_{n}\right\|_{E^{s}}+\left\|v_{n}\right\|_{E^{t}}\right)
$$

Thus, $\left\|u_{n}\right\|_{E^{s}}+\left\|v_{n}\right\|_{E^{t}}$ is bounded.

With this it is now possible to complete the proof of the (PS)-condition:

Since $\left\|u_{n}\right\|_{E^{s}}$ is bounded, we find a weakly convergent subsequence $u_{n} \rightarrow u$ in $E^{s}$. Since the mappings $A^{s}: E^{s} \rightarrow L^{2}$ and $A^{-t}: L^{2} \rightarrow E^{t}$ are continuous isomorphisms, we get $A^{s}\left(u_{n}-u\right) \rightarrow 0$ in $L^{2}$ and $A^{s-t}\left(u_{n}-u\right) \rightarrow 0$ in $E^{t}$. Furthermore, since $E^{t} \subset L^{q+1}$ compactly, we conclude that $A^{s-t}\left(u_{n}-u\right) \rightarrow 0$ strongly in $L^{q+1}$.

Similarly, we find a subsequence of $\left\{v_{n}\right\}$ which is weakly convergent in $E^{t}$ and such that $v_{n}^{q}$ is strongly convergent in $L^{\frac{q+1}{q}}$

Choosing $(\phi, \psi)=\left(0, A^{s-t}\left(u_{n}-u\right) \in E^{s} \times E^{t}\right.$ in (4.10) we thus conclude

$$
\int_{\Omega} A^{s} u_{n} A^{s}\left(u_{n}-u\right)=\int_{\Omega} v_{n}^{q} A^{s-t}\left(u_{n}-u\right)+\epsilon_{n}\left\|A^{s-t}\left(u_{n}-u\right)\right\|
$$


By the above considerations, the righthand-side converges to 0 , and thus

$$
\int_{\Omega}\left|A^{s} u_{n}\right|^{2} \rightarrow \int_{\Omega}\left|A^{s} u\right|^{2}
$$

Thus, $u_{n} \rightarrow u$ strongly in $E^{s}$.

To obtain the strong convergence of $\left\{v_{n}\right\}$ in $E^{t}$, one proceeds similarly: as above, one finds a subsequence $\left\{v_{n}\right\}$ converging weakly in $E^{t}$ to $v$, and then $A^{t-s} v_{n} \rightarrow A^{t-s} v$ weakly in $E^{s}$ and hence also in $L^{p+1}$, while by the above $u_{n} \rightarrow u$ strongly in $E^{s}$ and hence in $L^{p+1}$, and then $u_{n}^{p} \rightarrow u_{n}^{p}$ in $L^{\frac{p+1}{p}}$. Choosing in $(4.8)(\phi, \psi)=\left(A^{t-s}\left(v_{n}-v\right), 0\right)$, we get

$$
\int_{\Omega} A^{t}\left(v_{n}-v\right) A^{t} v_{n}=\int_{\Omega}\left|u_{n}\right|^{p-1} u_{n} A^{t-s}\left(v_{n}-v\right)+\epsilon_{n}\left(\left\|A^{t-s}\left(v_{n}-v\right)\right\|\right)
$$

and thus one concludes again that

$$
\int_{\Omega}\left|A^{t} v_{n}\right|^{2} \rightarrow \int_{\Omega}\left|A^{t} v\right|^{2}
$$

and hence also $v_{n} \rightarrow v$ strongly in $E^{t}$.

Thus, the conditions of Theorem 4.5 are satisfied; hence, we find a (positive) critical point $(u, v)$ for the functional $I$, which yields a weak solution to system (4.7).

\subsection{The $W^{1, \alpha}$ approach}

In this section we use that, alternatively, the functional $I(u, v)$ can be defined on a product of $W^{1, \alpha}$ spaces.

\subsubsection{The functional framework}

We define the functional $I(u, v)$ on a product space of Sobolev spaces. The term $\int_{\Omega} \nabla u \nabla v d x$ can be defined on the product space

$$
W_{0}^{1, \alpha}(\Omega) \times W_{0}^{1, \beta}(\Omega), \text { with } \frac{1}{\alpha}+\frac{1}{\beta}=1,
$$

by using the Hölder inequality

$$
\left|\int_{\Omega} \nabla u \nabla v d x\right| \leq\|\nabla u\|_{L^{\alpha}}\|\nabla v\|_{L^{\beta}}=\|u\|_{W_{0}^{1, \alpha}}\|v\|_{W_{0}^{1, \beta}}
$$

Thus, to have the terms $F(s)=\frac{1}{p+1}|s|^{p+1}$ and $G(s)=\frac{1}{q+1}|s|^{q+1}$ in $I(u, v)$ well defined, we need to impose, using the Sobolev embedding theorem:

$$
p+1 \leq \alpha^{*} \frac{\alpha N}{N-\alpha N} \quad, \quad q+1 \leq \beta^{*}=\frac{\beta N}{N-\beta N}
$$

From this we o obtain the condition

$$
\frac{1}{p+1}+\frac{1}{q+1} \geq \frac{N-\alpha}{\alpha N}+\frac{N-\beta}{\beta N}=1-\frac{2}{N} .
$$

Thus, we have found again the critical hyperbola. 


\section{Generalization to Orlicz spaces}

The $W^{1, \alpha}$ approach has the advantage that it can be generalized to more general settings by using Orlicz spaces. These spaces were already mentioned in section 4 when we discussed the Trudinger inequality. We discuss now some details about Orlicz spaces.

\subsection{Orlicz spaces}

We recall here some basic facts about Orlicz spaces, for more details see for instance $[1,32,44,26]$.

Definition 5.1 A continuous function $M: \mathbb{R} \rightarrow[0,+\infty)$ is called a $N$-function, if it is convex, even, $M(t)=0$ if and only if $t=0$, and

$$
M(t) / t \rightarrow 0 \text { as } t \rightarrow 0 \text { and } M(t) / t \rightarrow+\infty \text { as } t \rightarrow+\infty .
$$

Definition 5.2 Let $A$ and $B$ be $N$-functions. We say that

1) A dominates $B$ (near infinity) if, for some positive constant $k$,

$$
B(x) \leq A(k x) \text { for } x \geq x_{0}, \text { and write } B \prec A
$$

2) $A$ and $B$ are equivalent if $A$ dominates $B$ and $B$ dominates $A$; then we write $A \sim B$.

3) $B$ increases essentially more slowly than $A$ if

$$
\lim _{t \rightarrow \infty} \frac{B(k t)}{A(t)}=0, \text { for all } k>0
$$

in this case we write $B \prec \prec A$.

Associated to the N-function $M$ we introduce the following class of functions.

Definition 5.3 [Orlicz class] The Orlicz class is the set of functions defined by

$$
K_{M}(\Omega):=\left\{u: \Omega \rightarrow \mathbb{R}: u \text { measurable and } \int_{\Omega} M(u(x)) d x<\infty\right\} .
$$

Orlicz classes are convex sets, but in general not linear spaces. One then defines

Definition 5.4 [Orlicz space] The vector space $L_{M}(\Omega)$ generated by $K_{M}(\Omega)$ is called Orlicz space.

Proposition 5.1 The Orlicz class $K_{M}(\Omega)$ is a vector space, and hence equal to $L_{M}(\Omega)$ if and only if $M$ satisfies the following $\Delta_{2}$-condition: 
Definition $5.5\left(\Delta_{2}\right.$-condition) There exist numbers $k>1$ and $t_{0} \geq 0$ such that

$$
M(2 t) \leq k M(t), \text { for } t \geq t_{0}
$$

Furthermore, we define

Definition $5.6\left[\nabla_{2}\right.$-condition $]$ There exist numbers $h>1$ and $t_{1} \geq 0$ such that

$$
M(t) \leq \frac{1}{2 h} M(h t), \text { for } t \geq t_{1}
$$

We call a function satisfying the $\Delta_{2}-$ and the $\nabla_{2}$-condition $\Delta-$ regular.

We remark that the Orlicz class depends only on the asymptotic growth of the function $M$; therefore, also the $\Delta_{2}$-condition and the $\nabla_{2}$-condition need to be satisfied only near infinity.

We define the following norm on $L_{M}(\Omega)$ :

Definition 5.7 [Luxemburg norm]

$$
\|u\|_{(M)}=\inf \left\{\lambda>0: \int_{\Omega} M\left(\frac{|u|}{\lambda}\right) \leq 1\right\}
$$

Proposition $5.2\left(L_{M},\|\cdot\|_{(M)}\right)$ is a Banach space.

Definition 5.8 [Conjugate function] Let

$$
\widetilde{M}(x)=\sup _{y>0}\{x y-M(y)\}
$$

$\widetilde{M}$ is called the conjugate function of $M$.

It is clear that $\widetilde{\widetilde{M}}=M$, and $M$ and $\widetilde{M}$ satisfy the Young inequality:

$$
s t \leq M(t)+\widetilde{M}(s) \quad \forall s, t \in \mathbb{R}
$$

with equality when $s=M^{\prime}(t)$ or $t=\widetilde{M}^{\prime}(s)$.

Proposition 5.3 In the spaces $L_{M}$ and $L_{\widetilde{M}}$ the Hölder inequality holds:

$$
\left|\int_{\Omega} u(x) v(x) d x\right| \leq 2\|u\|_{(M)}\|v\|_{(\widetilde{M})}
$$


Hence, for every $\widetilde{u} \in L_{\widetilde{M}}$ we can define a continuous linear functional $l_{\widetilde{u}} v:=\int_{\Omega} \widetilde{u} v d x$ and $l_{\widetilde{u}} \in\left(L_{M}\right)^{*}$. Then we can define

$$
\|\tilde{u}\|_{\widetilde{M}}:=\left\|l_{\tilde{u}}\right\|=\sup _{\|v\|_{(M)} \leq 1} \int_{\Omega} \tilde{u}(x) v(x) d x
$$

Definition $5.9\|\tilde{u}\|_{\widetilde{M}}$ is called the Orlicz norm on the space $L_{\widetilde{M}}$, and analogously one defines the Orlicz norm $\|u\|_{M}$ on $L_{M}$.

Thus, we have two different norms on $L_{M}$, the Luxemburg (or gauge) norm $\|\cdot\|_{(M)}$ and the Orlicz norm $\|\cdot\|_{M}$; they are equivalent, and satisfy

$$
\|u\|_{(M)} \leq\|u\|_{M} \leq 2\|u\|_{(M)}
$$

In order to be precise about which norm is considered in the spaces, we are going to use from now on the following notations:

$$
\left(L_{M},\|\cdot\|_{M}\right):=L_{M} \text { and }\left(L_{M},\|\cdot\|_{(M)}\right):=L_{(M)},
$$

and similarly for $\widetilde{M}$.

It follows from the definition of Orlicz norm that

Proposition 5.4 If $u \in L_{M}$ and $\widetilde{w} \in L_{\widetilde{M}}$, then one has the following Hölder inequality

$$
\left|\int_{\Omega} u \widetilde{w} d x\right| \leq\|u\|_{M}\|\widetilde{w}\|_{(\widetilde{M})}
$$

Proposition 5.5 (see J.P. Gossez [26], Rao-Ren [44], p. 111)

$L_{M}$ is reflexive if and only if $M$ and $\widetilde{M}$ satisfy the $\Delta_{2}$ - condition, and then

$$
\left(L_{(M)}\right)^{*}=L_{\widetilde{M}} \quad \text { and }\left(L_{(\widetilde{M})}\right)^{*}=L_{M}
$$

Proposition 5.6 (see Rao-Ren [44], Thm 2, p. 297).

If $\Phi$ is $\Delta$-regular, then there exists a $\Phi_{1} \sim \Phi$ such that $L_{\Phi}=L_{\Phi_{1}}$ as sets, and their Luxemburg norms (respectively Orlicz norms) are equivalent, with the following additional structure:

a) $L_{\Phi}$ and $L_{\Phi_{1}}$ are isomorphic, and both are reflexive spaces

b) $L_{\Phi_{1}}$ is uniformly convex and uniformly smooth 
Next, we define the Orlicz-Sobolev spaces: Let $A$ be a N-function. Then set

\section{Definition:}

$$
W^{1} L_{A}=\left\{u: \Omega \rightarrow \mathbb{R} ; \max _{|\alpha| \in\{0,1\}} \int_{\Omega} A\left(\left|D^{\alpha} u\right|\right)<+\infty\right\}
$$

with Luxemburg norm

$$
\|u\|_{W^{1} L_{A}}:=\max \left\{\left\|D^{\alpha} u\right\|_{(A)}:|\alpha| \in\{0,1\}\right\}
$$

On the space $W_{0}^{1} L_{(A)}$, i.e. the space of functions in $W^{1} L_{A}$ which vanish on the boundary, an equivalent Luxemburg norm is given by

$$
\|u\|_{1,(A)}=\|\nabla u\|_{(A)}=\inf \left\{\lambda>0: \int_{\Omega} A\left(\frac{|\nabla u|}{\lambda}\right) \leq 1\right\} .
$$

The equivalence of these two norms is a consequence of the Poincaré inequality,

$$
\|u\|_{(M)} \leq C \sum_{i=1}^{n}\left\|D_{i} u\right\|_{(M)}, \forall u \in W_{0}^{1} L_{M}(\Omega),
$$

(see [26]). In analogy with the above definition of the Orlicz norm in $L_{M}$ we can define an Orlicz norm in $W_{0}^{1} L_{(A)}$ by

$$
\|u\|_{1, A}:=\sup \left\{\int_{\Omega} \nabla u \nabla \widetilde{w} d x: \widetilde{w} \in W_{0}^{1} L_{(\widetilde{A})},\|\widetilde{w}\|_{1,(\widetilde{A})} \leq 1\right\}
$$

The space $W_{0}^{1} L_{(A)}$ endowed with this new norm is denoted by $W_{0}^{1} L_{A}$.

Definition 5.10 [Sobolev conjugate] (see Adams [1], p. 248)

Suppose that $\int_{1}^{\infty} \frac{A^{-1}(t)}{t^{1+1 / n}} d t=+\infty$. Then the Sobolev conjugate function $\Phi(t)$ is given by

$$
\Phi^{-1}(t)=\int_{0}^{t} \frac{A^{-1}(\tau)}{\tau^{1+1 / n}} d \tau, t \geq 0 .
$$

Proposition 5.7 Let $\Omega$ be bounded, and satisfying the cone property. Then the embedding

$$
W^{1} L_{A}(\Omega) \hookrightarrow L_{\Phi}(\Omega)
$$

is continuous, and compact into $L_{G}(\Omega)$ where $G$ is any $N$-function increasing essentially more slowly than $\Phi$, i.e. $\lim _{t \rightarrow \infty} \frac{G(k t)}{\Phi(t)}=0$, for all $k>0$. 
Example 5.1 One easily checks that for $\Phi(s)=s^{p+1}$ the above formula (5.3) yields $A(s)=c s^{\alpha}$, with $\alpha$ satisfying $\frac{1}{\alpha}=\frac{1}{p+1}+\frac{1}{N}$; indeed, $\Phi^{-1}(t)=t^{1 /(p+1)}$, and hence

$$
\frac{A^{-1}(t)}{t^{1+\frac{1}{N}}}=\frac{d}{d t} \Phi^{-1}(t)=\frac{1}{p+1} t^{\frac{1}{p+1}-1}
$$

i.e.

$$
A^{-1}(t)=\frac{1}{p+1} t^{\frac{1}{p+1}+\frac{1}{N}}
$$

It follows that $p+1=\frac{\alpha N}{N-\alpha}$, and thus we we recover the classical Sobolev embedding theorem $W^{1, \alpha}(\Omega) \hookrightarrow L^{p+1}(\Omega)$.

Next, we make the following

Definition 5.11 Let $g \in C(\mathbb{R})$ be a $N$-function, and $G$ its primitive. Then we say that $G$ is $\theta$ - regular, if there exists a constant $\theta_{G}>1$ such that

$$
\lim _{s \rightarrow \infty} \frac{s g(s)}{G(s)}=\theta_{G}
$$

Let $F(t)=G^{-1}(t)$, and $f(t)=F^{\prime}(t)$. Then the above condition is equivalent to

$$
\lim _{t \rightarrow \infty} \frac{t f(t)}{F(t)}=\frac{1}{\theta_{G}}
$$

Indeed, we have $G(s)=t \Leftrightarrow F(t)=s$, and $f(t)=\frac{d}{d t}\left[G^{-1}(t)\right]=\frac{1}{g(s)}$.

We have

Proposition 5.8 (see Rao-Ren [44], p. 26) If $G$ is $\theta$ - regular, then $G$ is $\Delta$ regular, i.e. $G \in \Delta_{2} \cap \nabla_{2}$.

\subsection{Orlicz space criticality}

In the last section we have seen that Orlicz spaces are a generalization of $L^{p}$ spaces (which correspond to the N-functions $|s|^{p}$ ) to more general $N$-functions. It is natural to look for an analogue of the critical hyperbola for $N$-functions.

Definition: [critical Orlicz pair] Let $\Phi$ and $\Psi$ be $\Delta$-regular $N$-functions. Then $(\Phi, \Psi)$ are a critical Orlicz pair if there exist $\Delta$-regular and conjugate $N$ functions $A$ and $\widetilde{A}$ such that $L_{\Phi}$ and $L_{\Psi}$ are the smallest Orlicz spaces with

$$
W^{1} L_{A} \hookrightarrow L_{\Phi} \quad, \quad W^{1} L_{\widetilde{A}} \hookrightarrow L_{\Psi}
$$

We have the following: 
Theorem 5.9 Let $\Omega \subset \mathbb{R}^{N}$ be a bounded, smooth domain. Let $\Phi \in C^{1}$ be a given $N$-function, and set $\varphi(t)=\Phi^{\prime}(t)$. Assume that

$$
\lim _{s \rightarrow \infty} \frac{\varphi(s) s}{\Phi(s)}=\theta_{\Phi}>\frac{N}{N-2} .
$$

Then there exists an associated $N$-function $\Psi$ such that $(\Phi, \Psi)$ form a critical Orlicz pair. Furthermore, the limit

$$
\lim _{s \rightarrow \infty} \frac{s \Psi(s)}{\Psi(s)}=\theta_{\Psi}
$$

exists, and

$$
\frac{1}{\theta_{\Phi}}+\frac{1}{\theta_{\Psi}}=1-\frac{2}{N}
$$

Consider the following

Example 5.2 In Example 5.1 we saw that to $\Phi(s)=s^{p+1}$ corresponds the inverse Sobolev conjugate $A(s)=c s^{\alpha}$, with

$$
\frac{1}{p+1}+\frac{1}{N}=\frac{1}{\alpha} \text {. }
$$

The conjugate function $\widetilde{A}$ to $A$ is given by $\widetilde{A}(s)=c s^{\beta}$, with $\frac{1}{\alpha}+\frac{1}{\beta}=1$, which in turn has as Sobolev conjugate $\Psi(s)=s^{q+1}$, with

$$
\frac{1}{q+1}+\frac{1}{N}=\frac{1}{\beta}
$$

Adding the two equations yields

$$
\frac{1}{p+1}+\frac{1}{q+1}=1-\frac{2}{N} \text {. }
$$

This is the critical hyperbola, see de Figueiredo-Felmer [15] and HulshoffVanderVorst [28]. Thus, $\left(|s|^{p+1},|s|^{q+1}\right)$ are a critical Orlicz pair, and so the above theorem contains the critical hyperbola as a special case. We remark that the proof given here is also new in the polynomial case; in [15] and [28] fractional Sobolev spaces $H^{s}$ were used in order to conserve the Hilbert space structure.

We now give some examples of critical Orlicz pairs:

Example 5.3 Let

$$
\Phi(s) \sim|s|^{p+1}(\log |s|)^{\alpha} \quad \text { and } \quad \Psi(s) \sim|s|^{q+1}(\log |s|)^{-\alpha \frac{q+1}{p+1}},
$$

with $\alpha>0$ and $p, q \in(1,+\infty)$ satisfying (5.8). Then $\Phi$ and $\Psi$ satisfy (5.6), with $\theta_{\Phi}=p+1$ and $\theta_{\Psi}=q+1$, respectively, and $(\Phi, \Psi)$ form a critical Orliczpair; for the proof, see [20].

Remark 5.12 The restriction $\theta_{\Phi}>N /(N-2)$ in Theorme 5.9 is necessary in order to obtain a $\Psi$ which is $\theta$-regular, in the sense of Definition 5.11. Also in the polynomial case such a restriction, which here is $p+1>N /(N-2)$, is necessary in order to obtain $q>1$. 


\subsection{Critical Orlicz-pairs: proof of Theorem 5.9}

1) Hypothesis (5.6) expresses the fact that the function $\Phi$ is $\theta$-regular with $\theta_{\Phi}>N /(N-2)$. Let $A$ be the inverse Sobolev conjugate of $\Phi$, see Definition 5.10. Note that $W^{1} L_{A}$ is the largest Orlicz-Sobolev space that embeds into $L_{\Phi}$.

Claim 1: $A$ is $\theta$ - regular, with $\theta_{A}=\frac{N \theta_{\Phi}}{N+\theta_{\Phi}}>1$.

Indeed, let $F(s)=\Phi^{-1}(s)$ and $B(t)=A^{-1}(t)$. Then $F(s)=\int_{0}^{s} \frac{B(t)}{t^{1+1 / N}} d t$, and hence

$$
f(s)=\frac{B(s)}{s^{1+1 / N}} .
$$

Then we have by $(5.5)$

$$
\frac{1}{\theta_{\Phi}}=\lim _{s \rightarrow \infty} \frac{f(s) s}{F(s)}=\lim _{s \rightarrow \infty} \frac{B(s) s^{-1 / N}}{F(s)} .
$$

Then, by l'Hôpital's rule

$$
\lim _{s \rightarrow \infty} \frac{B(s) s^{-1 / N}}{F(s)}=\lim _{s \rightarrow \infty} \frac{b(s) s^{-1 / N}-\frac{1}{N} s^{-\frac{1}{N}-1} B(s)}{\frac{B(s)}{s^{1+1 / N}}}=\lim _{s \rightarrow \infty} \frac{b(s) s}{B(s)}-\frac{1}{N} .
$$

We conclude that

$$
\frac{1}{\theta_{\Phi}}=\lim _{s \rightarrow \infty} \frac{b(s) s}{B(s)}-\frac{1}{N}
$$

and thus

$$
\lim _{s \rightarrow \infty} \frac{b(s) s}{B(s)}=\frac{1}{\theta_{\Phi}}+\frac{1}{N}<1 .
$$

This implies that $A$ is $\theta$ - regular, with $\theta_{A}=\frac{N \theta_{\Phi}}{N+\theta_{\Phi}}>1$.

2) Next, let $\widetilde{A}$ be the conjugate function of $A$, given by definition $5.8 . \widetilde{A}$ is a $N$-function, and $\Delta$-regular, see Rao-Ren [44], Cor. 4, p. 26.

In the following, suppose that $s=A^{\prime}(t)$ (iff $t=\widetilde{A}^{\prime}(s)$ ); note that $t \rightarrow \infty$ iff $s \rightarrow \infty$. Then

$$
\frac{1}{\theta_{A}}=\lim _{t \rightarrow \infty} \frac{A(t)}{t A^{\prime}(t)}=\lim _{t \rightarrow \infty} \frac{A(t)}{t s}=\lim _{s \rightarrow \infty} \frac{s t-\widetilde{A}(s)}{s t}=1-\lim _{s \rightarrow \infty} \frac{\widetilde{A}(s)}{s \widetilde{A}^{\prime}(s)}=1-\frac{1}{\theta_{\widetilde{A}}}
$$

Thus, $\widetilde{A}$ is $\theta$ - regular, with $\theta_{\widetilde{A}}>1$.

We can now define the corresponding Orlicz-Sobolev space $W^{1} L_{\widetilde{A}}$.

3) Next, use definition 5.10 again to define the function $\Psi$; by Adams-Fournier [1], p. $248, \Psi$ is an $N$-function.

Claim 2: $\Psi$ is $\theta$ - regular, with $\theta_{\Psi}=\frac{N \theta_{\widetilde{A}}}{N-\theta_{\tilde{A}}}$.

This follows similarly as in claim 1, reversing the direction in the arguments. 
Finally, $L_{\Psi}$ is the smallest Orlicz space into which $W^{1} L_{\widetilde{A}}$ imbeds continuously.

Thus, we have shown that $(\Phi, \Psi)$ is a critical Orlicz pair.

Finally, we have

$$
\frac{1}{\theta_{\Phi}}+\frac{1}{\theta_{\Psi}}=\frac{N-\theta_{A}}{N \theta_{A}}+\frac{N-\theta_{\widetilde{A}}}{N \widetilde{A}}=\frac{1}{\theta_{A}}-\frac{1}{N}+\frac{1}{\theta_{\widetilde{A}}}-\frac{1}{N}=1-\frac{2}{N} .
$$

\subsection{Orlicz space subcritical: an existence theorem}

We have the following existence theorem for nonlinearities which have subcritical growth with respect to a given critical Orlicz pair $(\Phi, \Psi)$.

Theorem 5.13 Suppose that $(\Phi, \Psi)$ is a critical Orlicz-pair. Suppose that $f$ and $g$ are continuous functions, and let $F$ and $G$ denote their primitives. Assume that

(H1) there exists $\theta>2$ and $t_{0}>0$ such that for all $t \geq t_{0}$

$$
0<\theta F(t) \leq t f(t) \text { and } 0<\theta G(t) \leq t g(t)
$$

(H2) $F$ and $G$ are uniformly superquadratic near zero, i.e. there exist numbers $\sigma>2$ and $c \geq 1$ such that

$$
F(s t) \leq c s^{\sigma} F(t), G(s t) \leq c s^{\sigma} G(t), \forall t>0, \forall s \in[0,1]
$$

(H3) $F$ and $G$ have an essentially lower growth than $\Phi$ and $\Psi$, respectively (see Definition 5.2).

(H4) $F$ and $G$ satisfy

$$
\lim _{t \rightarrow 0} \frac{F(t)}{\Phi(t)}=C_{F}<\infty \text { and } \lim _{t \rightarrow 0} \frac{G(t)}{\Psi(t)}=C_{G}<\infty
$$

Then system (4.1) has a nontrivial solution.

Example 5.4 Let $(\Phi, \Psi)$ denote the critical Orlicz pair given in Example 5.3. Suppose that $F(s) \sim s^{p+1}(\log s)^{\beta}$ and $G(s) \sim s^{q+1}(\log s)^{-\gamma}$, for $s$ positive and large with $\beta<\alpha$ and $\gamma>\alpha \frac{q+1}{p+1}$. Then $F$ and $G$ have essentially slower growth than $\Phi$ and $\Psi$, respectively.

Proof. (outline; for more details, see [20])

We now define the functional

$$
I(u, \widetilde{v})=\int_{\Omega} \nabla u \nabla \widetilde{v} d x-\int_{\Omega} F(u) d x-\int_{\Omega} G(\widetilde{v}) d x
$$


on the space

$$
I: W_{0}^{1} L_{A}(\Omega) \times W_{0}^{1} L_{(\widetilde{A})}(\Omega) \rightarrow \mathbb{R}
$$

Here $\widetilde{v} \in W_{0}^{1} L_{(\widetilde{A})}(\Omega)$ is an independent variable; we write $\widetilde{v}$ to emphasize that $\widetilde{v}$ belongs to the space $W_{0}^{1} L_{(\widetilde{A})}(\Omega)$.

We recall that the functional (5.9) is strongly indefinite, being positive definite, resp. negative definite (near the origin) on an infinite dimensional subspace. We now make this more precise, by showing that there is the geometry of (local) infinite dimensional linking. For this, we introduce the

Tilde map: for $u \in W_{0}^{1} L_{A}(\Omega)$ consider

$$
S_{u}:=\sup \left\{\int_{\Omega} \nabla u(x) \nabla \widetilde{w}(x) d x: \widetilde{w} \in W_{0}^{1} L_{(\widetilde{A})},\|\widetilde{w}\|_{1,(\widetilde{A})}=\|u\|_{1, A}\right\} .
$$

Then we have

Lemma 5.14 There exists a unique $\widetilde{u} \in W_{0}^{1} L_{(\widetilde{A})}$ such that

$$
\|\widetilde{u}\|_{1,(\widetilde{A})}=\|u\|_{1, A} \quad \text { and } \quad S_{u}=\int_{\Omega} \nabla u(x) \nabla \tilde{u}(x) d x=\|u\|_{1, A}\|\widetilde{u}\|_{1,(\widetilde{A})}
$$

Furthermore, $\tilde{u}$ depends continuously (but nonlinearly) on $u$.

We now define two submanifolds of $E:=W_{0}^{1} L_{A}(\Omega) \times W_{0}^{1} L_{(\widetilde{A})}$ :

$$
E^{+}=\left\{(u, \tilde{u}): u \in W_{0}^{1} L_{A}\right\} \text { and } E^{-}=\left\{(u,-\widetilde{u}): u \in W_{0}^{1} L_{A}\right\}
$$

We note that $E^{+}$and $E^{-}$are nonlinear submanifolds of $E$ when regarded with respect to the standard vector space structure of $E$, but they are linear with respect to the following notion of

Tilde sum: given $(u, \widetilde{v}),(y, \widetilde{z}) \in E$, set

$$
(u, \widetilde{v}) \widetilde{+}(y, \widetilde{z}):=(u+y, \widetilde{v+z}) .
$$

One proves easily that with this notion one has

$$
E=E^{+} \widetilde{\oplus} E^{-}
$$

One then proves the following local linking structure:

\section{Lemma 5.15}

1) There exist $\rho_{0}, \sigma_{0}>0$ such that $I(z) \geq \sigma_{0}$, for all $z \in \partial B_{\rho_{0}} \cap E^{+}$.

2) There exist positive constants $R_{0}, R_{1}$ such that $I(z) \leq 0$ for all $z \in \partial Q$, where $Q=\left\{r\left(e_{1}, \widetilde{e_{1}}\right) \widetilde{+} w: w \in E^{-},\|w\| \leq R_{0}\right.$ and $\left.0 \leq r \leq R_{1}\right\} \quad\left(e_{1}\right.$ denotes the first eigenfunction of the Laplacian, with $\left.\left\|\left(e_{1}, \widetilde{e}_{1}\right)\right\|=1\right)$. 
We emphasize that this linking is only formal, since it involves two infinite dimensional manifolds. We now proceed by a finite dimensional approximation; this will lead to an actual linking structure. The proof is then completed by a limiting argument.

Define

$$
E_{n}^{+}:=\left\{(z, \widetilde{z}) ; z \in E_{n}\right\} \text { and } E_{n}^{-}:=\left\{(z,-\widetilde{z}) ; z \in E_{n}\right\},
$$

where $E_{n}$ is the space spanned by the first $n$ eigenfunctions of the Laplacian.

We now restrict the functional $I$ to $E_{n}^{+} \widetilde{\oplus} E_{n}^{-}=E_{n} \times \widetilde{E}_{n}$, and consider the set

$$
Q_{n}=\left\{w \widetilde{+} r\left(e_{1}, \widetilde{e}_{1}\right) ; w \in E_{n}^{1},\|w\| \leq R_{0}, 0 \leq r \leq R_{1}\right\} \subset E_{n}^{+} \widetilde{\oplus} E_{n}^{-} .
$$

Furthermore, we define the family of maps

$$
H_{n}=\left\{h \in C\left(Q_{n}, E_{n}^{+} \widetilde{E}_{n}^{-}\right): h(z)=z \quad \text { on } \partial Q_{n}\right\}
$$

One shows, using the topological degree on oriented manifolds, that

Lemma 5.16 The sets $Q_{n}$ and $\partial B_{\rho_{0}} \cap E_{n}^{+}$link, i.e.

$$
H\left(Q_{n}\right) \cap\left(\partial B_{\rho_{0}} \cap E_{n}^{+}\right) \neq 0, \forall h \in H_{n} .
$$

Finally, we set

$$
c_{n}=\inf _{h \in H_{n}} \max _{z \in Q_{n}} I(h(z)) .
$$

Using the linking property and Lemma 5.15 one obtains that the values $c_{n}$ satisfy $c_{n} \in\left[\sigma_{0}, c R_{1}^{2}\right], \forall n \in \mathbb{N}$. Furthermore, by the "Linking Theorem" of P.H. Rabinowitz [43] one obtains a PS-sequence $\left(u_{m}, \widetilde{v}_{m}\right) \in E_{n}^{+} \widetilde{\oplus} E_{n}^{-}$, i.e. satisfying $I\left(u_{m}, \widetilde{v}_{m}\right) \rightarrow c_{n}$, as $m \rightarrow \infty$, and $\left|I^{\prime}\left(u_{m}, \widetilde{v}_{m}\right)[(\xi, \widetilde{\eta})]\right| \leq \epsilon_{m}\|(\xi, \widetilde{\eta})\|$, with $\epsilon_{m} \rightarrow 0$ as $m \rightarrow \infty$.

One now shows

Lemma 5.10 Let $\left(u_{m}, \widetilde{v}_{m}\right)$ be a PS-sequence. Then the sequence $\left(u_{m}, \widetilde{v}_{m}\right)$ is bounded in $E$, i.e. there exists a constant $C$ such that $\left\|u_{m}\right\| \leq C$ and $\left\|\widetilde{v}_{m}\right\| \leq C$, for all $m \in \mathbb{N}$.

The proof of this Lemma uses standard methods, which are however rendered complicated by the fact that $L^{p}$ estimates must be replaced by more delicate estimates in Orlicz spaces.

With this, one now obtains that $c_{n}$ is a critical level of $\left.I\right|_{E_{n}^{+} \tilde{\oplus} E_{n}^{-}}$, for each $n \in \mathbb{N}$, with a corresponding sequence of critical points $z_{n} \in E_{n}^{+} \widetilde{\oplus} E_{n}^{-}$, with $\left\|z_{n}\right\| \leq c$, where $c$ does not depend on $n$. 
To complete the proof, we take the limit $n \rightarrow \infty$ : by the uniform bounds on $c_{n}$ and on the finite dimensional critical points $z_{n}$, it is easy to conclude that $z_{n}=\left(u_{n}, \widetilde{v}_{n}\right) \rightarrow z=(u, \widetilde{v})$ in $E=W_{0}^{1} L_{A} \times W_{0}^{1} L_{\widetilde{A}}$, and that $z$ is a weak solution of system (4.1).

It remains to show that $z=(u, \widetilde{v})$ is nontrivial; assume by contradiction that $u=0$, then by the equations (4.1) also $\widetilde{v}=0$. Note that we can find a suitable $\Delta$-regular N-function $F_{1}$ with $F_{1} \prec \prec \Phi$ and the properties $F(x) \leq F_{1}(x), f(x) \leq f_{1}(x), \forall x \in \mathbb{R}^{+}$. Thus, by the compact embedding $W_{0}^{1} L_{A} \hookrightarrow L_{F_{1}}$, we get

$$
\left\|u_{n}\right\|_{\left(F_{1}\right)} \rightarrow 0, \text { i.e. } \inf \left\{\lambda>0 ; \int_{\Omega} F_{1}\left(\frac{u_{n}}{\lambda}\right) \leq 1\right\}=: \lambda_{n} \rightarrow 0
$$

Since, for $\lambda_{n}<1$ holds $\frac{1}{\lambda_{n}} \int_{\Omega} F_{1}\left(u_{n}\right) \leq \int_{\Omega} F_{1}\left(\frac{u_{n}}{\lambda_{n}}\right) \leq 1$, we conclude that

$$
\int_{\Omega} F\left(u_{n}\right) \leq \int_{\Omega} F_{1}\left(u_{n}\right) \leq \lambda_{n} \rightarrow 0
$$

Since $F_{1}$ is $\Delta$-regular, we have $x f_{1}(x) \leq c F_{1}(x)$, for some $c>1$, and hence

$$
0 \leq \int_{\Omega} f\left(u_{n}\right) u_{n} \leq \int_{\Omega} f_{1}\left(u_{n}\right) u_{n} \leq c \int_{\Omega} F_{1}\left(u_{n}\right) d x \rightarrow 0
$$

It is easily seen that this implies that also $\int_{\Omega} \nabla u_{n} \nabla \widetilde{v}_{n} d x \rightarrow 0$, and thus also $I\left(u_{n}, \widetilde{v}_{n}\right) \rightarrow 0$. But this contradicts that $I\left(u_{n}, \widetilde{v}_{n}\right) \geq \sigma_{0}>0$, for all $n \in \mathbb{N}$.

This concludes the proof of Theorem 5.13.

In Theorem 5.13 we have obtained solutions for system (4.1) in the case of Orlicz-subcriticality; in particular, for the polynomial "model" system

$$
\left\{\begin{array}{rc}
-\Delta u=v^{q} & \text { in } \Omega \subset \mathbb{R}^{N}, N \geq 3 \\
-\Delta v=u^{p} & \text { on } \partial \Omega \\
u=v=0 &
\end{array}\right.
$$

this means that we have existence of solutions if the pair of nonlinearities $\left(|s|^{p+1},|s|^{q+1}\right)$ are Orlicz subcritical which, as we have seen, is in this case equivalent to say that the pair $(p, q)$ is below the critical hyperbola, i.e.

$$
\frac{1}{p+1}+\frac{1}{q+1}>1-\frac{2}{N}
$$




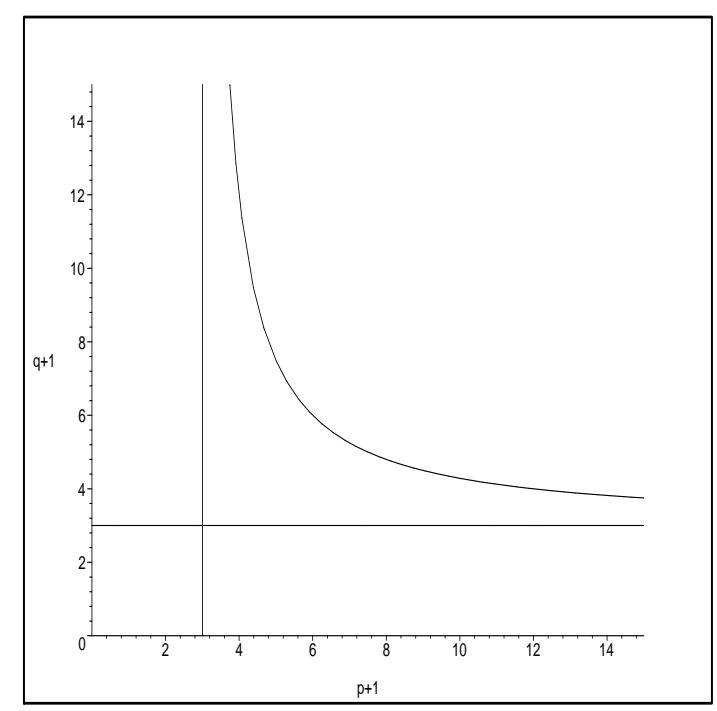

Figure 1: The critical hyperbola, $N=3$

\section{A system with no growth restriction in one nonlinearity}

We now turn our attention to some other aspects of the critical hyperbola. Note that the asymptotes of the critical hyperbola are given by

$$
\left(\frac{N}{N-2}, s\right), \text { and }\left(s, \frac{N}{N-2}\right), s \in \mathbb{R} .
$$

We show next that if one of the nonlinearities in system (4.1), , say $f(s)$, has a polynomial growth restriction with exponent lying to the left of the asymptote $\left(\frac{N}{N-2}, s\right)$ of the hyperbola, then no growth restriction on the other nonlinearity is needed. More precisely, restricting attention to the following model situation:

$$
\left\{\begin{aligned}
-\Delta u=g(v) & \\
-\Delta v=u^{p} & \text { in } \Omega \subset \mathbb{R}^{N}, \\
u=v=0 & \text { on } \partial \Omega
\end{aligned}\right.
$$

we have the following theorem, cf. [22]:

Theorem 6.1 Suppose that

$$
\begin{cases}0<p, & \text { if } N=2 \\ 0<p<\frac{2}{N-2}, & \text { if } N \geq 3\end{cases}
$$

and assume that $g \in C(\mathbb{R})$, with $G(s)=\int_{0}^{s} g(t) d t$ its primitive. Then the functional

$$
I(u, v)=\int_{\Omega} \nabla u \nabla v d x-\frac{1}{p+1} \int_{\Omega}|u|^{p+1} d x-\int_{\Omega} G(v) d x
$$


is well-defined and of class $C^{1}$ on the space

$$
E=E^{s}(\Omega) \times E^{t}(\Omega), \text { with } s \text { satisfying } p+1=\frac{2 N}{N-2 s}
$$

Proof. We proceed as in subsection 4.3 and define the equivalent functional (cf. (4.3))

$$
I(u, v)=\int_{\Omega} A^{s} u A^{t} v-\frac{1}{p+1} \int_{\Omega}|u|^{p+1} d x-\int_{\Omega} G(v) d x .
$$

The first term of the functional is naturally defined on the space $E$, and the second term is well-defined, continuous and differentiable, by the continuous embeddidng $E^{s} \subset L^{p+1}$, for $p+1=\frac{2 N}{N-2 s}$.

Next, note that

$$
t=2-s=2-\frac{N}{2}+\frac{N}{p+1}>2-\frac{N}{2}+N-2=\frac{N}{2}
$$

where we used the assumption $p+1<\frac{N}{N-2}$. Thus, we have the continuous embedding, cf. [1]

$$
E^{t}(\Omega) \subset C^{0, \frac{1}{2}}(\Omega),
$$

and hence we have for $v \in E^{t}$ that $v$ is continuous, and hence also $G(v)$, and then $\int_{\Omega} G(v) d x$ is well-defined, continuous and differentiable.

Next, we give an existence theorem for system (6.1). Since in this survey we concentrate on superlinear problems, i.e. with $p>1$, we obtain from $2<p+1<\frac{N}{N-2}$ the restriction $N \leq 3$. For corresponding results on "superlinear-sublinear" systems, we refer to [22]. Concerning system (6.1), with $N=2,3$, we have the following existence theorem

Theorem 6.2 Suppose that

$$
\begin{cases}0<p, & \text { if } N=2 \\ 0<p<\frac{2}{N-2}, & \text { if } N \geq 3\end{cases}
$$

and assume that $g \in C(\mathbb{R})$, with $G(s)=\int_{0}^{s} g(t) d t$ its primitive, and that there exist constants $\theta>2$ and $s_{0} \geq 0$ such that

$$
\theta G(s) \leq g(s) s, \forall|s| \geq s_{0} .
$$

Finally, for $s$ near 0 we assume $g(s)=o(s)$

Then system (6.1) has a nontrivial (strong) solution (u,v).

Proof. The proof follows closely the proof of Theorem 4.4, and we omit it. For details, we refer to [22]. 


\section{A borderline case}

In this section we will consider the borderline case where one of the nonlinearities has a growth corresponding to the asymptote $\left(\frac{N}{N-2}, s\right)$ of the critical hyperbola (5.8): we assume that

$$
F(s) \sim|s|^{\frac{N}{N-2}}
$$

and consider the system

$$
\left\{\begin{array}{rlrl}
-\Delta u=g(v) & \\
-\Delta v=u^{\frac{2}{N-2}} & & \text { in } \Omega \subset \mathbb{R}^{N}, \\
u=v=0 & & \text { on } \partial \Omega
\end{array}\right.
$$

We will see that then the nonlinearity $g(s)$ is governed by a limiting Sobolev space embedding, i.e. we find a maximal growth defined by a Trudinger-type embedding. However, there is a surprise!

We may again apply the $H^{s}$-approach or the $W^{1, \alpha}$-approach; and we will also consider a "mixed approach", defining the functional on $W^{s, \alpha}$-spaces, i.e. the space of functions whose fractional derivative of order $s$ lies in $L^{\alpha}$. We have seen that the $H^{s}$-approach and the $W^{1, \alpha}$-approach give the same critical hyperbola, and we will show that this is also the case for the "mixed approach". Thus, all these approaches seem equivalent. We will however show the surprising result that for the borderline case $F(s) \sim|s|^{\frac{N}{N-2}}$, these methods yield different maximal growth conditions for the nonlinearity $G(s)$. We will then use Lorentz spaces to obtain a truly maximal growth for $G(s)$.

\subsection{The $W^{s, \alpha}$-approach}

This is a "mixed" approach between the $H^{s}$ and the $W^{1, \alpha}$ approach. The space $W_{0}^{s, \alpha}$ consists of the functions whose fractional derivative of order $s$ lie in $L^{\alpha}$; a precise definition may be given by interpolation, see Adams-Fournier [1]. For these spaces, the usual Sobolev embedding theorems hold, see [1]; in particular, we have

$$
W_{0}^{s, \alpha} \subset L^{p+1}, \text { with } p+1=\frac{\alpha N}{N-s \alpha} .
$$

We define the functional $I(u, v)$ on the space

$$
W_{0}^{s, \alpha}(\Omega) \times W_{0}^{t, \beta}(\Omega), \text { with } s+t=2, \frac{1}{\alpha}+\frac{1}{\beta}=1
$$

As in section 4.3.1 we consider the functional

$$
I(u, v)=\int_{\Omega} A^{s} u A^{t} v-\frac{1}{p+1} \int_{\Omega}|u|^{p+1}-\frac{1}{q+1} \int_{\Omega}|v|^{q+1}
$$


The first term of the functional, $\int_{\Omega} A^{s} u A^{t} v d x$, is well defined on $W_{0}^{s, \alpha} \times W_{0}^{t, \beta}$ by estimating

$$
\left|\int_{\Omega} A^{s} u A^{t} v d x\right| \leq\|u\|_{W^{s, \alpha}}\|v\|_{W t, \beta}, \quad \text { with } s+t=2 \text { and } \frac{1}{\alpha}+\frac{1}{\beta}=1 .
$$

From the embeddings (7.2) we then get the following maximal growth conditions for $F(u)=\frac{1}{p+1}|u|^{p+1}$ and $G(v)=\frac{1}{q+1}|v|^{q+1}$ :

$$
p+1=\frac{\alpha N}{N-s \alpha}, q+1=\frac{\beta N}{N-s \beta}
$$

with the conditions:

$$
\frac{1}{p+1}+\frac{1}{q+1}=\frac{N-s \alpha}{\alpha N}+\frac{N-t \beta}{\beta N}=\frac{1}{\alpha}+\frac{1}{\beta}-\frac{s+t}{N}=1-\frac{2}{N} ;
$$

that is, we have found again the critical hyperbola!

\subsubsection{Different results using the $H^{s}$-method and the $W^{s, \alpha}$-method}

We now consider the border line case mentioned above: we assume that one of the nonlinearities has an exponent on the asymptote of the critical hyperbola, i.e.

$$
F(u)=\frac{1}{p+1}|u|^{p+1}=\frac{N-2}{N}|u|^{\frac{N}{N-2}} .
$$

We will see that the maximal growth for the other nonlinearity, $g(v)$, is given by a Trudinger-type inequality, i.e. it is of exponential growth. We will consider both, the $H^{s}$-approach and the $W^{s, \alpha}$ approach, and surprisingly, we will get different maximal growths!

As seen in section 4.4, the corresponding functional

$$
I(u, v)=\int_{\Omega} \nabla u \nabla v-\frac{N-2}{N} \int_{\Omega}|u|^{\frac{N}{N-2}}-\int G(v)
$$

can be defined on the space

$$
W_{0}^{1, \alpha}(\Omega) \times W_{0}^{1, \beta}(\Omega), \text { with } \frac{1}{\alpha}+\frac{1}{\beta}=1 .
$$

The requirement

$$
W^{1, \alpha}(\Omega) \subset L^{\frac{N}{N-2}}(\Omega)
$$

yields the condition

$$
\frac{N}{N-2}=\alpha^{*}=\frac{N \alpha}{N-\alpha} \Longleftrightarrow \frac{N}{N-1}=\alpha .
$$


By (7.3) this gives $\beta=N$, i.e. we are in the limiting Sobolev case $W^{1, \beta}(\Omega)=$ $W^{1, N}(\Omega)$. We look now for the largest possible growth $\phi(s)$ such that $\int_{\Omega} \phi(u) d x$ is finite. Indeed, by Trudinger [49] and Pohozaev [41] we have

$$
W^{1, N}(\Omega) \subset L_{\phi}(\Omega)
$$

where $L_{\phi}$ is the Orlicz space with the corresponding $N$-function

$$
\phi(s)=e^{|s|^{\frac{N}{N-1}}}-1 .
$$

Thus, with the $W^{1, \alpha}$-method we obtain as "critical growth" for the primitive $G(s)$ :

$$
G(s) \sim e^{|s|^{\frac{N}{N-1}}}
$$

i.e. we have the maximal growths

$$
F(s)=|s|^{p+1}=|s|^{\frac{N}{N-2}} \quad, \quad G(s) \sim e^{|s|^{\frac{N}{N-1}}}
$$

We now use the fractional Sobolev space approach: we work directly with the mixed approach, , i.e. the $W^{s, \alpha}$-method. Given $s \in(0,2)$, the optimal fractional Sobolev space $W_{0}^{s, \alpha}$ for having a continuous embedding $W_{0}^{s, \alpha} \subset L^{\frac{N}{N-2}}$ is given by the condition:

$$
\frac{N}{N-2}=p+1=\frac{\alpha N}{N-s \alpha}
$$

which implies

$$
\alpha=\frac{N}{N-(2-s)},
$$

and hence by $\frac{1}{\alpha}+\frac{1}{\beta}=1$ we get for $\beta$ the condition:

$$
\beta=\frac{N}{2-s}=\frac{N}{t} .
$$

The space $W_{0}^{t, \beta}(\Omega)=W_{0}^{t, \frac{N}{t}}(\Omega)$ is again a limiting Sobolev case, and by R.S. Strichartz [46] we have the optimal embedding

$$
W_{0}^{t, \beta}(\Omega)=W_{0}^{t, \frac{N}{t}}(\Omega) \subset L_{\phi}(\Omega)
$$

with

$$
\phi(r)=e^{|r|^{\frac{N}{N-t}}}-1, \quad 0<t<2 .
$$

Thus, the maximal growths by the $W^{s, \alpha}$-method are

$$
F(s)=|s|^{\frac{N}{N-2}}, \text { and } G(s) \sim e^{|s|^{\frac{N}{N-t}}}, 0<t<2,
$$

i.e. we have found a variable "critical growth" which depends on the choice of the value of $t$ !

We emphasize once more that we have found for system (7.1), by using the same functional $I(u, v)$, in changing function space settings, different maximal growths for the nonlinearity $G(s)$. This is of course somewhat disturbing! 


\subsection{Lorentz spaces}

We now change from Sobolev space settings to Lorentz spaces.

We recall the definition of a Lorentz space: For $\phi: \Omega \rightarrow \mathbb{R}$ a measurable function, we denote by

$$
\mu_{\phi}(t)=|\{x \in \Omega: \phi(x)>t\}| \quad, t \geq 0
$$

its distribution function. The decreasing rearrangement $\phi^{*}(s)$ of $\phi$ is defined by

$$
\phi^{*}(s)=\sup \left\{t>0 ; \mu_{\phi}(t)>s\right\}, 0 \leq s \leq|\Omega| .
$$

The Lorentz space $L(p, q)$ is defined as follows:

$$
\phi \in L(p, q), 1<p<\infty, 1 \leq q<\infty,
$$

if

$$
\|\phi\|_{p, q}=\left(\int_{0}^{\infty}\left[\phi^{*}(t) t^{1 / p}\right]^{q} \frac{d t}{t}\right)^{1 / q}<+\infty
$$

Lorentz spaces have the following main properties (see Adams-Fournier [1]):

1. $L(p, p)=L^{p}, 1<p<+\infty$

2. The following inclusions hold for $1<q<p<r<\infty$ :

$$
L^{r} \subset L(p, 1) \subset L(p, q) \subset L(p, p)=L^{p} \subset L(p, r) \subset L^{q}
$$

3. Hölder inequality:

$$
\left|\int_{\Omega} f g d x\right| \leq\|f\|_{p, q}\|g\|_{p^{\prime}, q^{\prime}}, \quad \text { where } p^{\prime}=\frac{p}{p-1}, q^{\prime}=\frac{q}{q-1}
$$

Furthermore, the following embedding theorems hold:

Theorem A: Suppose that $1 \leq p<N$, and that $\nabla u \in L(p, q)$; then $u \in L\left(p^{*}, q\right)$, where $p^{*}=\frac{N p}{N-p}$ and $1 \leq q<\infty$.

Note that this Theorem improves slightly Sobolev's embedding theorem, which gives $u \in L^{p^{*}}=L\left(p^{*}, p^{*}\right)$, which is a larger space than $L\left(p^{*}, p\right)$.

For the next theorem, see H. Brezis [9]:

Theorem B: Suppose that $u \in W^{j, p}$, with $p<\frac{N}{j}$; then $u \in L\left(p^{*}, p\right)$ with $\frac{1}{p^{*}}=\frac{1}{p}-\frac{j}{N}$.

The following theorem deals with the limiting Sobolev case, and is a generalization of Trudinger's result (see H. Brezis and S. Wainger [11], H. Brezis $[9])$. It is of particular importance for our considerations: 
Theorem C: Assume $\nabla u \in L(N, q)$ for some $1<q<\infty$. Then $e^{|u|^{\frac{q}{q-1}}} \in L^{1}$.

This generalizes the Trudinger embedding, which gives for $\nabla u \in L(N, N)$ that $e^{|u|^{\frac{N}{N-1}}} \in L^{1}$, i.e. the maximal growth $e^{|u|^{N-1}}$; we point out that in the BrezisWainger embedding the maximal growth depends only on the second Lorentz exponent $q$, but not on $N$.

We make the following

Definition Let $\Omega \subset \mathbb{R}^{N}$ be a bounded domain. Assume that $1<p<\infty$, $1<q<\infty$, and set

$$
W_{0}^{1} L(p, q)(\Omega)=\operatorname{cl}\left\{u \in C_{0}^{\infty}(\Omega):\|\nabla u\|_{p, q}<\infty\right\}
$$

On $W_{0}^{1} L(p, q)$ we have the following norm

$$
\|u\|_{1 ; p, q}:=\|\nabla u\|_{p, q}
$$

with which $W_{0}^{1} L(p, q)$ becomes a reflexive Banach space.

One now has the following sharpening of Theorem C, in analogy to Moser's sharpening of the Trudinger inequality.

Theorem D: There exists $\alpha_{0}=\alpha_{0}(N, p, \Omega)>0$ such that

$$
\sup _{\|\nabla u\|_{L(N, q)}=1} \int_{\Omega} e^{\alpha|u|^{\frac{q}{q-1}}}<+\infty, \text { for } \alpha \leq \alpha_{0}
$$

\subsection{Lorentz spaces and the asymptotic borderline case}

We consider again the functional

$$
I(u, v)=\int_{\Omega} \nabla u \nabla v d x-\int_{\Omega} G(v)-\frac{N-2}{N} \int_{\Omega}|u|^{\frac{N}{N-2}} d x .
$$

We want to consider the term $\int_{\Omega} \nabla u \nabla v d x$ on a product of Lorentz spaces, i.e. we want to estimate

$$
\left|\int_{\Omega} \nabla u \nabla v d x\right| \leq\|\nabla u\|_{L(p, q)}\|\nabla v\|_{L\left(p^{\prime}, q^{\prime}\right)},
$$

where we determine $p, q$ and $p^{\prime}, q^{\prime}$ such that the last term in $I(u, v)$ is welldefined, i.e.

$$
u \in L^{\frac{N}{N-2}}(\Omega)=L\left(\frac{N}{N-2}, \frac{N}{N-2}\right)(\Omega) .
$$

Thus, $q=\frac{N}{N-2}$, and by Theorem A we obtain the following condition for $p$

$$
\frac{N}{N-2}=p^{*}=\frac{N p}{N-p}
$$


and hence

$$
p=\frac{N}{N-1}
$$

Thus, we have to impose

$$
\nabla u \in L\left(\frac{N}{N-1}, \frac{N}{N-2}\right)
$$

Next, we calculate

$$
p^{\prime}=\frac{p}{p-1}=N \text { and } q^{\prime}=\frac{q}{q-1}=\frac{N}{2},
$$

and hence we get the condition

$$
\nabla v \in L\left(p^{\prime}, q^{\prime}\right)=L\left(N, \frac{N}{2}\right) .
$$

By Theorem $\mathrm{C}$ above we now find for $\nabla v \in L\left(N, \frac{N}{2}\right)$ that $e^{|v|^{\frac{N}{N-2}}} \in L^{1}(\Omega)$.

Thus we have

Theorem 7.1 The functional

$$
I(u, v)=\int_{\Omega} \nabla u \nabla v d x-\int_{\Omega} G(v)-\frac{N-2}{N} \int_{\Omega}|u|^{\frac{N}{N-2}} d x
$$

is well defined on the space

$$
W_{0}^{1} L\left(\frac{N}{N-1}, \frac{N}{N-2}\right)(\Omega) \times W_{0}^{1} L\left(N, \frac{N}{2}\right)(\Omega),
$$

and the maximal growth for $G(s)$ is given by

$$
G(s) \sim e^{|v|^{\frac{N}{N-2}}} .
$$

We remark that the growth (7.6) is considerably larger than the growth $G(s) \sim e^{|s|^{\frac{N}{N-1}}}$ found by the $H^{s}$-method in (7.4), and it corresponds to the limiting case $t \rightarrow 2$ in the $W^{s, \alpha}$-method (which however cannot be reached in that framework).

\subsection{Subcritical with respect to the asymptotic borderline case: existence of solution}

In this section we prove the existence of solutions for systems (7.1) in the "subcritical case". Again, since we are interested in the superlinear case, we restrict to dimension $N=3$, that is, we consider the system

$$
\begin{cases}-\Delta u=g(v) & \\ -\Delta v=u^{2} & \text { in } \Omega \subset \mathbb{R}^{3}, \\ u=v=0 & \text { on } \partial \Omega\end{cases}
$$


As mentioned, the corresponding functional is

$$
I(u, v)=\int_{\Omega} \nabla u \nabla v-\int_{\Omega} \frac{1}{3}|u|^{3}-\int_{\Omega} G(v)
$$

on the space

$$
E:=W_{0}^{1} L\left(\frac{3}{2}, 3\right) \times W_{0}^{1} L\left(3, \frac{3}{2}\right)
$$

and we assume that $g(s)$ has a subcritical growth, i.e. an essentially lower growth than $e^{|s|^{3}}$. More precisely, we assume that

$$
\lim _{s \rightarrow+\infty} \frac{g(s)}{e^{\alpha|s|^{3}}}=0, \forall \alpha>0
$$

Furthermore, we make the following assumptions on $g$ (our aim is to give simple assumptions, at the expense of greater generality):

A1) $g$ is a continuous function, with $g(s)=o(s)$ near the origin

A2) There exist constants $\nu>2$ and $s_{0}>0$ such that

$$
0<\nu G(s) \leq s g(s), \forall|s|>0 \text {. }
$$

A3) There exist constants $s_{1}>0$ and $M>0$ such that

$$
0<G(s) \leq M g(s) \text { for all }|s| \geq s_{1} .
$$

\section{Example}

$$
G(s)=e^{|s|^{\beta}}-1-|s|^{\beta} \text {, with } 0<\beta<3 .
$$

Theorem 7.1 , see [45]. Under assumptions A1) - A3) and (7.9), system (7.1) has a nontrivial positive (weak) solution $(u, v) \in E$.

Proof. The proof follows ideas from section 5.3. We remark that the proof is similar to one in section 9.2 below, and therefore we limit here to give an outline of the main idea.

First, note that the functional $I$ given in (7.8) is strongly indefinite. In order to overcome this difficulty, we introduce, as in section 5.4, a suitable "tilde map":

\section{a) The tilde map}

Consider the bilinear map $\int_{\Omega} \nabla u \nabla v d x$ on the space $W_{0}^{1} L\left(\frac{3}{2}, 3\right) \times W_{0}^{1} L\left(3, \frac{3}{2}\right)$. For $u \in W_{0}^{1} L\left(\frac{3}{2}, 3\right)$ denote with $\widetilde{u} \in W_{0}^{1} L\left(3, \frac{3}{2}\right)$ the unique element such that

$$
\sup _{\left\{v \in W_{0}^{1} L\left(3, \frac{3}{2}\right):\|\nabla v\|_{3, \frac{3}{2}}=\|\nabla u\|_{\frac{3}{2}, 3}\right\}} \int_{\Omega} \nabla u \nabla v d x=\int_{\Omega} \nabla u \nabla \widetilde{u} d x=\|\nabla u\|_{\frac{3}{2}, 3}\|\nabla \widetilde{u}\|_{3, \frac{3}{2}}
$$

and hence

$$
\int_{\Omega} \nabla u \nabla \widetilde{u} d x=\|\nabla u\|_{\frac{3}{2}, 3}^{2}=\|\nabla \widetilde{u}\|_{3, \frac{3}{2}}^{2}
$$


The existence and uniqueness of $\tilde{u}$ follows from the reflexivity and convexity of $W_{0}^{1} L\left(\frac{3}{2}, 3\right)$, see [20].

We can thus define the "tilde-map": $W_{0}^{1} L\left(\frac{3}{2}, 3\right) \rightarrow W_{0}^{1} L\left(3, \frac{3}{2}\right), u \mapsto \widetilde{u}$, which is continuous and positively homogeneous

On the product space $E=W_{0}^{1} L\left(\frac{3}{2}, 3\right) \times W_{0}^{1}\left(3, \frac{3}{2}\right)$ we now define two continuous submanifolds

$$
E^{+}=\left\{(u, \widetilde{u}): u \in W_{0}^{1} L\left(\frac{3}{2}\right), 3\right\}, \quad E^{-}=\left\{(u,-\widetilde{u}): u \in W_{0}^{1} L\left(\frac{3}{2}, 3\right)\right\}
$$

As remarked in [20], the nonlinear manifolds $E^{+}$and $E^{-}$have a linear structure with respect to the following notion of tilde-sum:

$$
(u, \widetilde{v}) \widetilde{+}(y, \widetilde{z}):=(u+y, \widetilde{v+z}),
$$

and one has

$$
E=E^{+} \widetilde{\oplus} E^{-}, \text {with norm }\|w\|_{E}^{2}=\|(u, \widetilde{v})\|_{E}^{2}=\|\nabla u\|_{3, \frac{3}{2}}^{2}+\|\nabla \widetilde{v}\|_{\frac{3}{2}, 3}^{2}
$$

\section{b) Linking structure}

Next, one verifies that the functional $I$ has a "linking structure" in the origin. This is proved similarly as in the corresponding proof in subsection 9.2 below, and we thus omit it here. For details, we refer to [45].

\section{c) Palais-Smale sequences are bounded}

Let $\left(u_{n}, \widetilde{v}_{n}\right) \in E$ be a Palais-Smale sequence for the functional $I$, i.e. such that with $\left|I\left(u_{n}, \widetilde{v}_{n}\right)\right| \leq d$, and $\left|I^{\prime}\left(u_{n}, \widetilde{v}_{n}\right)[(\phi, \widetilde{\psi})]\right| \leq \varepsilon_{n}\|(\phi, \widetilde{\psi})\|_{E}, \varepsilon_{n} \rightarrow 0, \forall(\phi, \widetilde{\psi}) \in$ $E$. Then $\left\|\left(u_{n}, \widetilde{v}_{n}\right)\right\|_{E} \leq c$.

Again, the proof is similar to subsection 9.2 below.

\section{d) Finite-dimensional approximation}

Since the functional $I$ is strongly indefinite on the space $E$ (i.e. positive and negative definite on infinite dimensional manifolds), the standard linking theorems cannot be applied. We therefore proceed by finite dimensional approximations (Galerkin procedure):

Denote by $\left(e_{i}\right)_{i \in \mathbb{N}}$ an orthonormal set of eigenfunctions corresponding to the eigenvalues $\left(\lambda_{i}\right), i \in \mathbb{N}$, of the Laplacian, with Dirichlet boundary conditions. Set

$$
E_{n}^{+}=\operatorname{span}\left\{\left(e_{i}, \widetilde{e}_{i}\right) \mid i=1, \ldots, n\right\}, E_{n}^{-}=\operatorname{span}\left\{\left(e_{i},-\widetilde{e}_{i}\right) \mid i=1, \ldots, n\right\}
$$

and

$$
E_{n}=E_{n}^{-} \widetilde{\oplus} E_{n}^{-}
$$

Exploiting the local linking structure given in b), it is now standard to conclude that for each $n \in \mathbb{N}$ the functional $I_{n}:=\left.I\right|_{E_{n}}$ has a critical point $z_{n}=\left(u_{n}, \widetilde{v}_{n}\right) \in E_{n}$ at level $c_{n}$, with

$$
I\left(z_{n}\right)=c_{n} \in\left[\sigma_{0}, \sigma_{1}\right], \sigma_{i} 0, i=1,2,
$$


and $I^{\prime}\left(z_{n}\right)[(\phi, \widetilde{\psi})]=0$, for all $(\phi, \widetilde{\psi}) \in E_{n}$. Hence we have

$$
\left\{\begin{array}{l}
\int_{\Omega} \nabla u_{n} \nabla \widetilde{\psi}=\int_{\Omega} g\left(\widetilde{v}_{n}\right) \widetilde{\psi} \\
\int_{\Omega} \nabla \widetilde{v}_{n} \nabla \phi=\int_{\Omega} u_{n}^{2} \phi
\end{array} \quad, \forall(\phi, \widetilde{\psi}) \in E_{n}\right.
$$

e) Limit $n \rightarrow \infty$ :

It remains to pass to the limit $n \rightarrow \infty$. By c) we have that $\left\|\left(u_{n}, \widetilde{v}_{n}\right)\right\|_{E} \leq c$, and hence $\left(u_{n}, \widetilde{v}_{n}\right) \rightarrow(u, \widetilde{v})$ in $E$. Furthermore, we may assume that

$$
\widetilde{v}_{n} \rightarrow \widetilde{v} \text { in } L^{\alpha}, \text { for all } \alpha \geq 1 \text {; }
$$

indeed, by the properties 1) and 2) of Lorentz spaces, see subsection 7.2 , we have the following continuous embeddings

$$
W_{0}^{1} L\left(3, \frac{3}{2}\right) \subset W_{0}^{1} L(3-\delta, 3-\delta)=W_{0}^{1,3-\delta} \subset L^{\frac{(3-\delta) N}{N-(3-\delta)}}=L^{\frac{(3-\delta) 3}{\delta}}, \text { for } \delta>0,
$$

and hence we have compact embedding into $L^{\alpha}$, for all $1 \leq \alpha<\frac{(3-\delta) 3}{\delta}$.

Proceeding as in subsection 9.2 below, one concludes that by taking the limit $n \rightarrow \infty$

$$
\left\{\begin{array}{l}
\int_{\Omega} \nabla u \nabla \widetilde{\psi}=\int_{\Omega} g(\widetilde{v}) \widetilde{\psi} \\
\int_{\Omega} \nabla \widetilde{v} \nabla \phi=\int_{\Omega} u^{2} \phi
\end{array} \quad, \forall(\phi, \widetilde{\psi}) \in \cup E_{n}=E,\right.
$$

i.e. $(u, \widetilde{v}) \in E$ is a (weak) solution of (7.14).

Finally, we prove that $(u, \widetilde{v}) \in E$ is nontrivial. If we assume to the contrary that $u=0$, then by (7.14) also $\widetilde{v}=0$. Since $g$ is subcritical, we obtain by (7.9) that for all $\delta>0$

$$
|g(t)| \leq c_{\delta} e^{\delta|t|^{3}}, \forall t \in \mathbb{R}
$$

Now we choose $\widetilde{\psi}=\widetilde{v}_{n}$ in the first equation of (7.14), and estimate by Hölder

$$
\left|\int_{\Omega} g\left(\widetilde{v}_{n}\right) \widetilde{v}_{n}\right| \leq c_{\delta}\left\|e^{\delta\left|\widetilde{v}_{n}\right|^{3}}\right\|_{L^{\beta}}\left\|\widetilde{v}_{n}\right\|_{L^{\alpha}} \leq d_{\delta}\left\|\widetilde{v}_{n}\right\|_{L^{\alpha}}
$$

where we have used that $\left\|\nabla \widetilde{v}_{n}\right\|_{3, \frac{3}{2}} \leq c$, and hence by Theorem $\mathrm{C}$ above, for $\beta>1$ sufficiently small:

$$
\left\|e^{\delta\left|\widetilde{v}_{n}\right|^{3}}\right\|_{L^{\beta}}=\int_{\Omega} e^{\delta \beta\left|\widetilde{v}_{n}\right|^{3}} \leq c .
$$

Since by (7.13) $\left\|\widetilde{v}_{n}\right\|_{L^{\alpha}} \rightarrow 0$, and hence by (7.15) $\int_{\Omega} g\left(\widetilde{v}_{n}\right) \widetilde{v}_{n} \mid \rightarrow 0$, we conclude by the first equation in (7.12), by multiplication by $\widetilde{v}_{n}$ and inegration, that

$$
\int_{\Omega} \nabla u_{n} \nabla \widetilde{v}_{n}=\int_{\Omega} g\left(\widetilde{v}_{n}\right) \widetilde{v}_{n} \rightarrow 0 .
$$


This in turn implies, by choosing $\phi=u_{n}$ in the second equation in (7.12), that also $\int_{\Omega}\left|u_{n}\right|^{3} \rightarrow 0$, and by assumption A2) follows that also $\int_{\Omega} G\left(\widetilde{v}_{n}\right) \rightarrow 0$. This implies finally that $I\left(u_{n}, \widetilde{v}_{n}\right)=\int_{\Omega} \nabla u_{n} \nabla \widetilde{v}_{n}-\int_{\Omega}\left(\frac{1}{3}\left|u_{n}\right|^{3}+G\left(\widetilde{v}_{n}\right)\right) \rightarrow 0$; but this contradicts $(7.11)$, and thus $(u, \widetilde{v}) \neq(0,0)$.

This completes the proof.

\section{Critical phenomena for the system}

As pointed out for the scalar equation, critical growth is connected with several interesting phenomena. We now discuss these properties for the system

$$
\left\{\begin{array}{c}
-\Delta u=v^{q+1} \\
-\Delta v=u^{p+1} \quad, \quad \text { in } \Omega \\
u=v=0 \quad, \quad \text { on } \partial \Omega
\end{array}\right.
$$

\subsection{Critical growth: non-existence of (positive) solutions}

First we present a result of E. Mitidieri [34] (see also van der Vorst [50]) which gives non-existence of positive solutions for system (4.1) if the nonlinearities have critical growth.

Theorem 8.1 Let $\Omega \subset \mathbb{R}^{N}$ be a starshaped domain. Assume that

$$
\frac{1}{p+1}+\frac{1}{q+1} \leq 1-\frac{2}{N}
$$

Then system (8.1) has no positive solution.

Proof. The proof relies on a Pohozaev type identity which is a modification of an identity by Pucci-Serrin [42].

Lemma 8.2 (see collorally 2.1 and (2.5) in [34])

Let $(u, v)$ (with $u, v \in C^{2}(\bar{\Omega})$ ) be a solution of system (8.1). Then

$$
\begin{aligned}
& \int_{\Omega}(\Delta u(x, \nabla v)+\Delta v(x, \nabla u)) d x \\
= & \int_{\partial \Omega}\left(\frac{\partial u}{\partial n}(x, \nabla v)+\frac{\partial v}{\partial n}(x, \nabla u)-(\nabla u, \nabla v)(x, n)\right) d s \\
& +(N-2) \int_{\Omega}(\nabla u, \nabla v) d x
\end{aligned}
$$


Applying this identity to system (8.1) we obtain

$$
\begin{aligned}
& N \int_{\Omega}\left(\frac{1}{p+1}|u|^{p+1}+\frac{1}{q+1}|v|^{q+1}\right) d x \\
& =(N-2) \int_{\Omega}(\nabla u, \nabla v) d x+\int_{\partial \Omega} \frac{\partial u}{\partial n} \frac{\partial v}{\partial n}(x, n) d s
\end{aligned}
$$

On the other hand, multiplying the first equation of (8.1) by $v$ and the second by $u$ and integrating, we obtain, for any $a \in(0,1)$

$$
\int_{\Omega} \nabla u \nabla v=a \int_{\Omega}|v|^{q+1} d x+(1-a) \int_{\Omega}|u|^{p+1} d x
$$

Now choose

$$
a=\frac{N}{(N-2)(p+1)}
$$

and hence by $(8.2)$

$$
1-a \geq \frac{N}{(N-2)(q+1)}
$$

which yields

$$
(N-2) \int_{\Omega}(\nabla u, \nabla v) d x \geq N \int_{\Omega}\left(\frac{1}{p+1}|u|^{p+1}+\frac{1}{q+1}|v|^{q+1}\right) d x
$$

Hence, by (8.4), we get

$$
0 \geq \int_{\partial \Omega} \frac{\partial u}{\partial n} \frac{\partial v}{\partial n}(x, n) d s
$$

Since $u$ and $v$ are positive solutions, we have by the maximum principle that

$$
\frac{\partial u}{\partial n}<0 \text { and } \frac{\partial v}{\partial n}<0 \text { on } \partial \Omega
$$

and since $(x, n)>0$ by the assumption that $\Omega$ is starshaped, we obtain a contradiction.

\subsection{Critical growth: non-compactness and concentration}

We discuss further "phenomena of critical growth" for the model system (8.1). We first remark that by "solving" the first equation for $v$ (assuming that $v>0$ ) we get $v=(-\Delta u)^{1 / q}$, and then we obtain by the second equation

$$
\left\{\begin{array}{l}
-\Delta\left((-\Delta u)^{1 / q}\right)=u^{p}, \quad \text { in } \Omega \\
u=\Delta u=0, \text { on } \partial \Omega
\end{array}\right.
$$

which is equivalent to system (8.1). 
The variational formulation for equation (8.6) leads to the minimization problem

$$
\inf _{u \in S_{p+1}} \int_{\Omega}|\Delta u|^{\frac{q+1}{q}} d x
$$

where

$$
S_{p+1}=\left\{u \in W^{2, \frac{q+1}{q}}(\Omega) \mid u=\Delta u=0 \text { on } \partial \Omega,\|u\|_{L^{p+1}}=1\right\}
$$

The Sobolev embedding theorem gives in this case

$$
W^{2, \frac{q+1}{q}}(\Omega) \subset L^{p+1}(\Omega), \text { for } p+1 \leq \frac{\frac{q+1}{q} N}{N-2 \frac{q+1}{q}}
$$

which is equivalent to

$$
\frac{1}{p+1}+\frac{1}{q+1} \geq 1-\frac{2}{N}
$$

i.e. the critical hyperbola!

We note that by Theorem 8.1 the infimum in (8.7) cannot be attained in starshaped domains, since otherwise there would exist a solution to system (8.1).

To understand the concentration behavior of minimizing sequences, it is important to study the situation in $\mathbb{R}^{N}$, as described in the case of the Laplacian in section 2.4. One has the following result by P.L. Lions [36], which is proved by the methods of concentration-compactness:

Theorem 8.3 Let

$$
m=\inf \left\{\left.\int_{\mathbb{R}^{N}}|\Delta u|^{\frac{q+1}{q}} d x\left|u \in \mathcal{D}^{2, \frac{q+1}{q}}, \int_{\mathbb{R}^{N}}\right| u\right|^{p+1}=1\right\},
$$

where $\mathcal{D}^{2, \frac{q+1}{q}}$ is the completion of $C_{0}^{\infty}\left(\mathbb{R}^{N}\right)$ in the norm $\|\Delta \cdot\|_{\frac{q+1}{q}}$. Then every minimizing sequence $\left(u_{n}\right)$ of $(8.8)$ is relatively compact in $\mathcal{D}^{2, \frac{q+1}{q}}$ up to translation and dilation, i.e. there exist $\left(y_{n}\right) \in \mathbb{R}^{N},\left(\sigma_{n}\right) \in(0, \infty)$ such that the new minimizing sequence

$$
\widetilde{u}_{n}=\sigma_{n}^{-N /(p+1)} u_{n}\left(\cdot-y_{n} / \sigma_{n}\right)
$$

is relatively compact in $\mathcal{D}^{2, \frac{q+1}{q}}$. In particular, there exists a minimum of (8.8).

\subsection{Critical growth: instantons}

The fact that the infimum $m$ in (8.8) is attained, and that there is a dilation invariance, says that we have again a family of instantons, given by the minimizers of (8.8). However, in contrast to the case of the Laplacian described in section 2.4, these instantons are not explicitly known. In [28] Hulshof and van der Vorst show that the minimizer (ground state) of (8.8) is (up to translation 
dilation) unique; furthermore, they show that the ground state is positive, radially symmetric and decreasing in $r$. Thus, all ground states of (8.8) are given by

$$
u_{\epsilon, x_{0}}=\epsilon^{-\frac{N}{p+1}} u\left(\frac{x-x_{0}}{\epsilon}\right)
$$

where $u$ is the unique "normalized" ground state with $u(0)=1$. Furthermore, Hulshof and van der Vorst derive the precise asymptotic behavior of the normalized ground state.

\subsection{Brezis-Nirenberg type results for systems}

In subsection 2.4 we have discussed the famous result of Brezis-Nirenberg [10], in which it is shown that the existence of solutions for elliptic equation with critical growth terms can be recovered by adding a suitable lower order perturbation. In the proof it is crucial to have the explicit form of the instantons in order to prove by explicit estimates that due to the perturbation the non-compactness level of the functional is avoided.

Hulshof, Mitidieri and van der Vorst [30] consider the corresponding problem for the following perturbed critical system

$$
\left\{\begin{aligned}
-\Delta u & =\mu v+v^{q+1} \\
-\Delta v & =\lambda u+u^{p+1} \quad, \quad \text { in } \Omega \\
u & =v=0 \quad, \quad \text { on } \partial \Omega
\end{aligned}\right.
$$

with

$$
\frac{1}{p+1}+\frac{1}{q+1}=1-\frac{2}{N}
$$

They give (rather complicated) conditions on $\mu$ and $\lambda$ such that system (8.9) has nontrivial solutions. The proof is based on the dual variational method, cf. Ekeland-Temam [13]. To show that the non compactness levels of the corresponding functional are avoided, they use the asymptotic behavior of the normalized ground state mentioned above.

\section{Systems in two dimensions}

We have seen in section 3 that critical growth for the scalar equation in dimension $N=2$ is governed by the Trudinger embedding and is of exponential type. For systems in two dimensions, one would like to obtain in analogy to the critical hyperbola in $N \geq 3$ a "critical curve" describing the maximal growth for the combined nonlinearities. This can be obtained using again Lorentz spaces. 


\subsection{Exponential Critical hyperbola}

In this section we assume that $\Omega \subset \mathbb{R}^{2}$ is a bounded domain, and consider again the system

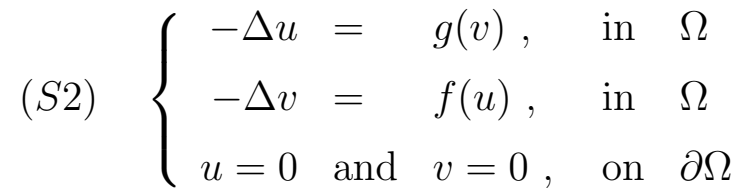

For the scalar equation $-\Delta u=f(u)$ in $\Omega, u=0$ on $\partial \Omega$, critical growth is given by the Trudinger-Moser inequlity, i.e. $F(u) \sim e^{|u|^{2}}$. For the system, we look for an analogue of the critical hyperbola for $N \geq 3$. By considering the functional

$$
J(u, v)=\int_{\Omega} \nabla u \nabla v d x-\int_{\Omega} F(u) d x-\int_{\Omega} G(v) d x
$$

on the space $H_{0}^{1} \times H_{0}^{1}$ one sees (as in the scalar case) that the nonlinearities $G(v) \sim e^{|v|^{2}}$ and $F(u) \sim e^{|u|^{2}}$ lie on this "critical curve". We assume now that $F(t)$ and $G(t)$ have "exponential polynomial growth", i.e.

$$
F(t) \sim e^{|t|^{p}}, \text { and } G(t) \sim e^{|t|^{q}}, \text { for some } 1<p, q<+\infty .
$$

We look for a relation between $p$ and $q$ such that the pair $(F, G)$ becomes critical. We prove:

Theorem 9.1 Let $\Omega \subset \mathbb{R}^{2}$ be a bounded domain. Then we have an "exponential critical curve" given by

$$
(F(s), G(s))=\left(e^{|s|^{p}}, e^{|s|^{q}}\right), \text { with } \frac{1}{p}+\frac{1}{q}=1 .
$$

Remark 9.2 One might try, as in the case $N \geq 3$, to work with spaces like $W^{1, \alpha} \times W^{1, \beta}$, with $\frac{1}{\alpha}+\frac{1}{\beta}=1$ and e.g. $0<\alpha<2$. However, note that then

$$
W^{1, \alpha}(\Omega) \subset L^{r}(\Omega), \text { with } r=\frac{\alpha N}{N-\alpha},
$$

and furthermore, since $\beta=\frac{\alpha}{\alpha-1}>2$, we get

$$
W_{0}^{1, \beta}(\Omega) \subset L^{\infty}(\Omega)
$$

i.e. we find a maximal growth of polynomial type for $F$,

$$
|F(s)| \leq|s|^{\frac{N \alpha}{N-\alpha}}
$$

and no growth restriction on the nonlinearity $G(s)$. So, this choice of spaces brings us for any $(\alpha, \beta) \neq(2,2)$ immediately outside the range of exponential nonlinearities.

Thus, to treat the problem, we need to work with spaces which lie "very close" to the space $W^{1,2}$, that is, we look for an "interpolation space" which lies between $W^{1,2}$ and $W^{1,2+\delta}$, for every $\delta>0$. We have introduced such a class of spaces in subsection 7.2, namely the Lorentz spaces. 
Proof. We consider the functional

$$
J(u, v)=\int_{\Omega} \nabla u \nabla v d x-\int_{\Omega} F(u)-\int_{\Omega} G(v)
$$

We want to consider the term $\int_{\Omega} \nabla u \nabla v d x$ on a product of Lorentz spaces, i.e. we want to estimate, using the Hölder inequality on Lorentz spaces:

$$
\left|\int_{\Omega} \nabla u \nabla v d x\right| \leq\|\nabla u\|_{L(2, q)}\|\nabla v\|_{L\left(2, q^{\prime}\right)}, \frac{1}{q}+\frac{1}{q^{\prime}}=1
$$

By Theorem C, section 7.2, we have that

$$
u \in W^{1} L(2, q) \Rightarrow e^{|u|^{\frac{q}{q-1}}} \in L^{1}, \quad \text { and } \quad v \in W^{1} L\left(2, q^{\prime}\right) \Rightarrow e^{|v|^{\frac{q^{\prime}}{q^{\prime}}-1}} \in L^{1} .
$$

Thus the maximal growth allowed for $F(s)=\int_{0}^{s} f(t) d t$ and $G(s)=\int_{0}^{s} g(t) d t$ is given by

$$
F(u) \sim e^{|u|^{p}}, p=q^{\prime}=\frac{q}{q-1}, \text { and } G(v) \sim e^{|v|^{q}}
$$

\subsection{Existence for the subcritical problem in dimension $N=2$}

In this section we show that for subcritical growth with respect to the exponential critical hyperbola defined in Theorem 9.1 we have compactness, and hence existence of solutions. We make the following assumptions on $f$ and $g$ (our aim is to give simple assumptions, at the expense of greater generality):

A1) $f$ and $g$ are continuous functions, with $f(s)=o(s)$ and $g(s)=o(s)$ near the origin

A2) There exist constants $\mu>2, \nu>2$ and $s_{0}>0$ such that

$$
0<\mu F(s) \leq s f(s) \text {, and } 0<\nu G(s) \leq s g(s), \forall|s|>0 \text {. }
$$

A3) There exist constants $s_{1}>0$ and $M>0$ such that

$$
\begin{aligned}
& 0<G(s) \leq M g(s) \text { for all }|s| \geq s_{1} \\
& 0<F(s) \leq M f(s) \text { for all }|s| \geq s_{1}
\end{aligned}
$$

We remark that assumption $A 3$ ) implies that $f$ and $g$ have at least exponential growth.

A4) $f$ has at most critical growth, i.e. there exist constants $a_{1}, a_{2}$ and $d$ such that

$$
f(s) \leq a_{1}+a_{2} e^{d|s|^{p}}
$$


A5) $g$ is subcritical, i.e. for all $\delta>0$ holds:

$$
\lim _{|s| \rightarrow \infty} \frac{g(s)}{e^{\delta|s|^{q}}}=0 \quad, \quad q=\frac{p}{p-1}
$$

\section{Example:}

$$
F(s)=e^{|s|^{p}}-1-|s|^{p}, G(s)=e^{|s|^{\beta}}-1-|s|^{\beta}, \text { with } 1<\beta<q .
$$

We consider the space $E=W_{0}^{1} L(2, q) \times W_{0}^{1} L(2, p)$.

Theorem 9.3 Under assumptions A1) - A5), system (S2) has a nontrivial positive (weak) solution $(u, v) \in E$.

Proof. The proof follows the lines of subsection 5.4 (see also [19] and [20]).

We note that the functional $J$ given in (9.1) is strongly indefinite in the origin, being positive and negative definite on infinite dimensional subspaces. If working on a Hilbert space $H$, one can find suitable subspaces $H^{+} \oplus H^{-}=H$ such that $\left.I\right|_{H^{+}}$is positive definite and $\left.J\right|_{H^{-}}$is negative definite near the origin. Since we are working on the Banach space $E$, the subspaces $H^{+}, H^{-}$have to be replaced by infinite dimensional manifolds. As in (5.10) we define

\section{a) The tilde map}

Consider the bilinear map $\int_{\Omega} \nabla u \nabla v d x$ on the space $W_{0}^{1} L(2, q) \times W_{0}^{1} L(2, p)$, where $p=\frac{q}{q-1}$.

For $u \in W_{0}^{1} L(2, q)$ denote with $\widetilde{u} \in W_{0}^{1} L(2, p)$ the unique element such that

$$
\sup _{\left\{v \in W_{0}^{1} L(2, p):\|\nabla v\|_{2, p}=\|\nabla u\|_{2, q}\right\}} \int_{\Omega} \nabla u \nabla v d x=\int_{\Omega} \nabla u \nabla \widetilde{u} d x=\|\nabla u\|_{2, q}\|\nabla \widetilde{u}\|_{2, p}
$$

and hence

$$
\int_{\Omega} \nabla u \nabla \widetilde{u} d x=\|\nabla u\|_{2, q}^{2}=\|\nabla \widetilde{u}\|_{2, p}^{2}
$$

The existence and uniqueness of $\widetilde{u}$ follows from the reflexivity and convexity of $W_{0}^{1} L(2, q)$, see [20].

We can thus define the "tilde-map": $W_{0}^{1} L(2, q) \rightarrow W_{0}^{1} L(2, p), u \mapsto \widetilde{u}$. This map is clearly continuous; it is nonlinear, but positively homogeneous: $\widetilde{\rho u}=\rho \widetilde{u}$, for all $\rho \geq 0$.

On the product space $E=W_{0}^{1} L(2, q) \times W_{0}^{1}(2, p)$ we can now define two continuous submanifolds

$$
E^{+}=\left\{(u, \widetilde{u}): u \in W_{0}^{1} L(2, q)\right\}, \quad E^{-}=\left\{(u,-\widetilde{u}): u \in W_{0}^{1} L(2, q)\right\}
$$

As remarked in Lemma 5.14 , the nonlinear manifolds $E^{+}$and $E^{-}$have a linear structure with respect to the following notion of tilde-sum:

$$
(u, \widetilde{v}) \widetilde{+}(y, \widetilde{z}):=(u+y, \widetilde{v+z}),
$$


and one has

$$
E=E^{+} \widetilde{\oplus} E^{-}, \text {with norm }\|w\|_{E}^{2}=\|(u, \widetilde{v})\|_{E}^{2}=\|\nabla u\|_{2, q}^{2}+\|\nabla \widetilde{v}\|_{2, p}^{2}
$$

\section{b) Linking structure}

Next, we verify that the functional $J(u, v)$ has a linking structure in $(0,0)$ :

i) Using A1) and A4) one estimates, for given $\epsilon>0$

$$
F(s) \leq \epsilon s^{2}+c|s|^{3} e^{d|s|^{p}}, G(s) \leq \epsilon s^{2}+c|s|^{3} e^{d|s|^{q}}
$$

Claim 1: There exist $\rho>0$ and $\sigma>0$ such that $J(u, \widetilde{u}) \geq \sigma$ for $\|(u, \widetilde{u})\|_{E}=\rho$; indeed, using (7.10) and

$$
\begin{aligned}
& J(u, \widetilde{u})=\int_{\Omega} \nabla u \nabla \widetilde{u}-\int_{\Omega} F(u)-\int_{\Omega} G(\widetilde{u}) \\
& \geq \frac{1}{2}\|\nabla u\|_{2, q}^{2}-\varepsilon \int_{\Omega}|u|^{2}-c\|u\|_{6}^{3}\left(\int_{\Omega} e^{2 d|u|^{p}}\right)^{1 / 2}+ \\
& +\frac{1}{2}\|\nabla \widetilde{u}\|_{2, p}^{2}-\varepsilon \int_{\Omega}|\widetilde{u}|^{2}-c\|\tilde{u}\|_{6}^{3}\left(\int_{\Omega} e^{2 d|\tilde{u}|^{q}}\right)^{1 / 2} .
\end{aligned}
$$

Now, we use that by Theorem D, subsection 7.2

$$
\begin{gathered}
\|u\|_{6} \leq d_{3}\|\nabla u\|_{2, q}, \text { and } \int_{\Omega} e^{2|u|^{p}} \leq c, \text { if }\|\nabla u\|_{2, q} \leq \theta_{1} \\
\|\widetilde{u}\|_{6} \leq d_{4}\|\nabla \widetilde{u}\|_{2, p}, \text { and } \int_{\Omega} e^{2|\widetilde{u}|^{q}} \leq c, \text { if }\|\nabla \widetilde{u}\|_{2, p} \leq \theta_{2}
\end{gathered}
$$

With these estimates the claim follows easily.

Next, fix $e_{1} \in W_{0}^{1} L(2, q)$ and $\widetilde{e}_{1} \in W_{0}^{1} L(2, p)$ with $\left\|\nabla e_{1}\right\|_{2, q}=\left\|\nabla \widetilde{e}_{1}\right\|_{2, p}=1$, and let

$$
Q=\left\{r\left(e_{1}, \widetilde{e}_{1}\right) \widetilde{+} w ; w \in E^{-},\|w\|_{E} \leq R_{0}, 0 \leq r \leq R_{1}\right\}
$$

Claim 2: There exist $R_{0}, R_{1}>0$ such that $J(z) \leq 0, \forall z \in \partial Q$, where $\partial Q$ denotes the boundary of $Q$ in $\mathbb{R}\left(e_{1}, \widetilde{e}_{1}\right) \widetilde{+} E^{-}$.

i) for $(u, \widetilde{v}) \in \partial Q \cap E^{-}$we have $(u, \widetilde{v})=(u,-\widetilde{u})$ and hence

$$
J(u,-\widetilde{u})=-\int_{\Omega} \nabla u \nabla \widetilde{u}-\int F(u)-\int G(-\widetilde{u}) \leq-\|\nabla u\|_{2, q}^{2} \leq 0
$$

ii) Let $(u, \widetilde{v})=r\left(e_{1}, \widetilde{e}_{1}\right) \tilde{+}(w,-\widetilde{w})=\left(r e_{1}+w, \widetilde{r e_{1}-w}\right) \in \partial Q$, with $\|(w,-\widetilde{w})\|_{E}=R_{0}, 0 \leq r \leq R_{1}$. 
First set $R_{1}=1$. Then

$$
\begin{aligned}
J(u, \widetilde{v}) & \leq \int_{\Omega} \nabla\left(r e_{1}+w\right) \nabla\left(\widetilde{r e_{1}-w}\right) \\
& =\int_{\Omega} \nabla\left(w-r e_{1}\right) \nabla\left(\widetilde{w-r e_{1}}\right)-\int_{\Omega} \nabla\left(2 r e_{1}\right) \nabla\left(\widetilde{w-r e_{1}}\right) \\
& \leq-\left\|\nabla\left(w-r e_{1}\right)\right\|_{2, q}^{2}+2\left\|\nabla r e_{1}\right\|_{2, q}\left\|\nabla\left(\widetilde{w-r e_{1}}\right)\right\|_{2, p} \\
& \leq-\|\nabla w\|_{2, q}^{2}-\left\|\nabla r e_{1}\right\|_{2, q}^{2}+2\|\nabla w\|_{2, q}\left\|\nabla r e_{1}\right\|_{2, q}+2\left\|\nabla r e_{1}\right\|_{2, q}\left(\|\nabla w\|_{2, q}+\left\|\nabla r e_{1}\right\|_{2, q}\right) \\
& \leq-\|\nabla w\|_{2, q}^{2}+4 r\|\nabla w\|_{2, q}+r^{2} \leq 0,
\end{aligned}
$$

for $2\|\nabla w\|_{2, q}^{2}=\|\nabla w\|_{2, q}^{2}+\|\nabla \widetilde{w}\|_{2, p}^{2}=\|(w,-\widetilde{w})\|_{E}^{2}=\bar{R}_{0}^{2}$ sufficiently large.

Note that this estimate now holds for all $\rho \geq 1$, with $0 \leq r \leq \rho$ and $\|(w,-\widetilde{w})\|_{E}^{2}=\rho \bar{R}_{0}$.

iii) Let $z=\rho\left(e_{1}, \widetilde{e}_{1}\right) \widetilde{+} \rho(w,-\widetilde{w}) \in \partial Q$, with $\|(w,-\widetilde{w})\|_{E} \leq \bar{R}_{0}$. Then by A2), for $\theta=\min \{\mu, \nu\}>2$

$$
\begin{aligned}
J(u, \widetilde{v}) & =\int_{\Omega} \nabla\left(\rho e_{1}+\rho w\right) \nabla\left(\widetilde{e_{1}-\rho w}\right)-\int_{\Omega} F\left(\rho e_{1}+\rho w\right)+G\left(\widetilde{e_{1}-\rho w}\right) \\
& \leq \rho^{2}\left\|\nabla\left(e_{1}+w\right)\right\|_{2, q}\left\|\nabla\left(e_{1}-w\right)\right\|_{2, q}-c \int_{\Omega}\left|\rho e_{1}+\rho w\right|^{\theta}+c_{1}-c \int_{\Omega}\left|\widetilde{\rho e_{1}-\rho} w\right|^{\theta}+c_{1} \\
& \leq \rho^{2}\left(\left\|\nabla e_{1}\right\|_{2, q}+\|\nabla w\|_{2, q}\right)^{2}-c \rho^{\theta}\left\{\int_{\Omega}\left|e_{1}+w\right|^{\theta}+\left.\iint_{\Omega} \widetilde{e_{1}-w}\right|^{\theta}\right\}+2 c_{1}
\end{aligned}
$$

It follows that

$$
J(u, \widetilde{v}) \leq \rho^{2}\left(1+\bar{R}_{0}\right)^{2}-c \rho^{\theta} \delta_{0}+2 c_{1} \leq 0
$$

for $\rho \geq R_{1}$ sufficiently large, where

$$
\delta_{0}=\inf _{\|(w,-\widetilde{w})\|_{E} \leq \bar{R}_{0}}\left\{\int_{\Omega}\left|e_{1}+w\right|^{\theta}+\int_{\Omega}\left|\widetilde{e_{1}-w}\right|^{\theta}\right\}>0 ;
$$

indeed, if $\delta_{0}=0$ we would find a sequence $w_{n}$ with $\left\|\left(w_{n},-\widetilde{w}_{n}\right)\right\| \leq \bar{R}^{0}$ and $\int_{\Omega}\left|e_{1}+w_{n}\right|^{\theta}+\left|\widetilde{e_{1}-w_{n}}\right|^{\theta} \rightarrow 0$. By the compact embeddings $W^{1} L(2, q) \subset L^{\theta}$ and $W^{1} L(2, p) \subset L^{\theta}$ we get strongly convergent subsequences $e_{1}+w_{n} \rightarrow e_{1}+w=0$ and $\widetilde{e_{1}-w_{n}} \rightarrow \widetilde{e_{1}-w}=0$, i.e. $w=e_{1}$ and $w=-e_{1}$ : contradiction.

Finally, defining $R_{0}=R_{1} \bar{R}_{0}$, the claim holds.

\section{c) Palais-Smale sequences are bounded}

Let $\left(u_{n}, \widetilde{v}_{n}\right) \in E$ with $\left|J\left(u_{n}, \widetilde{v}_{n}\right)\right| \leq d$, and

$$
\left|J^{\prime}\left(u_{n}, \widetilde{v}_{n}\right)[(\phi, \widetilde{\psi})]\right| \leq \varepsilon_{n}\|(\phi, \widetilde{\psi})\|_{E}, \varepsilon_{n} \rightarrow 0, \forall(\phi, \widetilde{\psi}) \in E
$$

Then $\left\|\left(u_{n}, \widetilde{v}_{n}\right)\right\|_{E} \leq c$. 
Indeed, choosing $(\phi, \widetilde{\psi})=\left(u_{n}, \widetilde{v}_{n}\right)=z_{n}$ in (9.4) we get, using A2)

$$
\begin{aligned}
\int_{\Omega} f\left(u_{n}\right) u_{n}+\int_{\Omega} g\left(\widetilde{v}_{n}\right) \widetilde{v}_{n} & \leq 2\left|\int_{\Omega} \nabla u_{n} \nabla \widetilde{v}_{n}\right|+\varepsilon_{n}\left\|\left(u_{n}, \widetilde{v}_{n}\right)\right\|_{E} \\
& \leq 2 d+2 \int_{\Omega} F\left(u_{n}\right)+2 \int_{\Omega} G\left(\widetilde{v}_{n}\right)+\varepsilon_{n}\left\|\left(u_{n}, \widetilde{v}_{n}\right)\right\|_{E} \\
& \leq 2 d+\frac{2}{\mu} \int_{\Omega} f\left(u_{n}\right) u_{n}+\frac{2}{\nu} \int_{\Omega} g\left(\widetilde{v}_{n}\right) \widetilde{v}_{n}+\varepsilon_{n}\left\|\left(u_{n}, \widetilde{v}_{n}\right)\right\|_{E}
\end{aligned}
$$

from which we get

$$
\int_{\Omega} f\left(u_{n}\right) u_{n} \leq c+\varepsilon_{n}\left\|\left(u_{n}, \widetilde{v}_{n}\right)\right\|_{E}, \int_{\Omega} g\left(\widetilde{v}_{n}\right) \widetilde{v}_{n} \leq c+\varepsilon_{n}\left\|\left(u_{n}, \widetilde{v}_{n}\right)\right\|_{E}
$$

and then also

$$
\int_{\Omega} F\left(u_{n}\right) \leq c, \int_{\Omega} G\left(\widetilde{v}_{n}\right) \leq c .
$$

Next, taking $(\phi, \widetilde{\psi})=\left(v_{n}, 0\right)$ and $(\phi, \widetilde{\psi})=\left(0, \widetilde{u}_{n}\right)$ in (9.4) we have

$$
\left\|\nabla v_{n}\right\|_{2, q}^{2} \leq \int_{\Omega} f\left(u_{n}\right) v_{n}+\varepsilon_{n}\left\|\left(v_{n}, 0\right)\right\|_{E},
$$

and

$$
\left\|\nabla \widetilde{u}_{n}\right\|_{2, p}^{2} \leq \int_{\Omega} g\left(\widetilde{v}_{n}\right) \widetilde{u}_{n}+\varepsilon_{n}\left\|\left(0, \widetilde{u}_{n}\right)\right\|_{E}
$$

Setting $V_{n}=\frac{v_{n}}{\left\|\nabla v_{n}\right\|_{2, q}}$ and $\widetilde{U}_{n}=\frac{\widetilde{u}_{n}}{\left\|\nabla \widetilde{u}_{n}\right\|_{2, p}}$ we obtain

$$
\left\|\nabla v_{n}\right\|_{2, q} \leq \int_{\Omega} f\left(u_{n}\right) V_{n}+\varepsilon_{n} \text { and }\left\|\nabla \widetilde{u}_{n}\right\|_{2, p} \leq \int_{\Omega} g\left(\widetilde{v}_{n}\right) \widetilde{U}_{n}+\varepsilon_{n} .
$$

We now use the following inequality: for any $\alpha>1$ (and setting $\alpha^{\prime}=\frac{\alpha}{\alpha-1}$ ) holds:

$$
s t \leq \begin{cases}\left(e^{t^{\alpha}}-1\right)+s\left(\log ^{+} s\right)^{1 / \alpha}, & \text { for all } t \geq 0 \text { and } s \geq e^{\left(\frac{1}{\alpha}\right)^{\alpha^{\prime}}} \\ \left(e^{t^{\alpha}}-1\right)+\frac{\alpha-1}{\alpha^{\alpha^{\prime}}} s^{\alpha^{\prime}}, & \text { for all } t \geq 0 \text { and } 0 \leq s \leq e^{\left(\frac{1}{\alpha}\right)^{\alpha^{\prime}}}\end{cases}
$$

\section{Proof}

For fixed $s>0$, consider $\sup _{t \geq 0}\left\{s t-\left(e^{t^{\alpha}}-1\right)\right\}$, and let $t_{s}$ denote the (unique) point where the supremum is attained; then $s=\alpha t_{s}^{\alpha-1} e^{t_{s}^{\alpha}}$.

i) $t_{s} \geq\left(\frac{1}{\alpha}\right)^{\frac{1}{\alpha-1}}$ : then $s=\alpha t_{s}^{\alpha-1} e^{t_{s}^{\alpha}} \geq e^{t_{s}^{\alpha}}$ and hence $(\log s)^{\frac{1}{\alpha}} \geq t_{s}$, and then

$$
\sup _{t \geq 0}\left\{s t-\left(e^{t^{\alpha}}-1\right)\right\}=s t_{s}-\left(e^{t_{s}^{\alpha}}-1\right) \leq s t_{s} \leq s(\log s)^{1 / \alpha}
$$


ii) $0 \leq t_{s} \leq\left(\frac{1}{\alpha}\right)^{\frac{1}{\alpha-1}}$ and $s \geq e^{\left(\frac{1}{\alpha}\right)^{\alpha^{\prime}}}$ : then $s t_{s} \leq s\left(\frac{1}{\alpha}\right)^{\frac{1}{\alpha-1}} \leq s\left(\log ^{+} s\right)^{\frac{1}{\alpha}}$, by the assumption on $s$.

iii) $0 \leq t_{s} \leq\left(\frac{1}{\alpha}\right)^{\frac{1}{1-\alpha}}$ and $s \leq e^{\left(\frac{1}{\alpha}\right)^{\alpha^{\prime}}}$ : in fact, the second inequality in II) holds always, since by I) $s t \leq t^{\alpha}+\frac{\alpha-1}{\alpha^{\alpha^{\prime}}} s^{\alpha^{\prime}} \leq\left(e^{t^{\alpha}}-1\right)+\frac{\alpha-1}{\alpha^{\alpha^{\prime}}} s^{\alpha^{\prime}}$, for all $s, t \geq 0$.

We apply the above inequality to the estimates in (9.9).

Applying inequality (9.10) with $\alpha=p$ and $t=\left|V_{n}(x)\right|, s=\left|f\left(u_{n}(x)\right)\right|$, to the first estimate in (9.9):

$$
\begin{aligned}
\left\|\nabla v_{n}\right\|_{2, q} & \leq \int_{\Omega} f\left(u_{n}\right) V_{n}+\varepsilon_{n} \\
& \leq \int_{\Omega}\left(e^{\left|V_{n}\right|^{p}}-1\right)+\int_{\Omega}\left|f\left(u_{n}\right)\right|\left[\log ^{+}\left|f\left(u_{n}\right)\right|\right]^{1 / p}+\varepsilon_{n} \\
& \leq c+\int_{\Omega} f\left(u_{n}\right) u_{n}+\varepsilon_{n} \\
& \leq c+\varepsilon_{n}+\varepsilon_{n}\left\|\left(u_{n}, \widetilde{v}_{n}\right)\right\|_{E}
\end{aligned}
$$

Applying inequality (9.10) with $\alpha=q$ and $t=\left|\widetilde{U}_{n}\right|, s=\left|g\left(v_{n}\right)\right|$, to the second estimate in (9.9), and using A5) and (9.5) yields

$$
\begin{aligned}
\left\|\nabla \widetilde{u}_{n}\right\|_{2, p} & \leq \int_{\Omega} g\left(\widetilde{v}_{n}\right) \widetilde{U}_{N}+\varepsilon_{n} \\
& \leq \int_{\Omega}\left(e^{\widetilde{U}_{n}^{q}}-1\right)+\int_{\Omega}\left|g\left(\widetilde{v}_{n}\right)\right|\left[\log ^{+}\left|g\left(\widetilde{v}_{n}\right)\right|\right]^{1 / q}+\varepsilon_{n} \\
& \leq c+\int_{\Omega} g\left(\widetilde{v}_{n}\right) \widetilde{v}_{n}+\varepsilon_{n} \\
& \leq c+\varepsilon_{n}\left\|\left(u_{n}, \widetilde{v}_{n}\right)\right\|_{E}+\varepsilon_{n}
\end{aligned}
$$

Joining the two inequalities yields the boundedness of $\left\|\left(u_{n}, \widetilde{v}_{n}\right)\right\|_{E}$.

\section{d) Finite-dimensional approximation}

Note that the functional $J$ is strongly indefinite on the space $E$ (i.e. positive and negative definite on infinite dimensional manifolds), and hence the standard linking theorems cannot be applied. We therefore consider an approximate problem on finite-dimensional spaces (Galerkin approximation):

Denote by $\left(e_{i}\right)_{i \in \mathbb{N}}$ an orthonormal set of eigenfunctions corresponding to the eigenvalues $\left(\lambda_{i}\right), i \in \mathbb{N}$, of $\left(-\Delta, H_{0}^{1}(\Omega)\right)$, and set

$$
\begin{aligned}
& E_{n}^{+}=\operatorname{span}\left\{\left(e_{i}, \widetilde{e}_{i}\right) \mid i=1, \ldots, n\right\} \\
& E_{n}^{-}=\operatorname{span}\left\{\left(e_{i},-\widetilde{e}_{i}\right) \mid i=1, \ldots, n\right\} \\
& E_{n}=E_{n}^{-} \widetilde{\oplus} E_{n}^{-}
\end{aligned}
$$


Set now $Q_{n}=Q \cap E_{n}, Q$ as in b) above, and define the family of mappings

$$
\Gamma_{n}=\left\{\gamma \in C\left(Q_{n}, E_{n}^{-} \widetilde{\oplus}\left[\left(e_{1}, \widetilde{e}_{1}\right)\right]\right) \mid \gamma(z)=z \text { on } \partial Q_{n}\right\}
$$

and set

$$
c_{n}=\inf _{\gamma \in \Gamma_{n}} \max _{z \in Q_{n}} J(\gamma(z)) .
$$

It is now quite standard (see [43], [20]) to conclude that:

Proposition 9.4 For each $n \in \mathbb{N}$ the functional $J_{n}=\left.J\right|_{E_{n}}$ has a critical point $z_{n}=\left(u_{n}, \widetilde{v}_{n}\right) \in E_{n}$ at level $c_{n}$, with

$$
J\left(z_{n}\right)=c_{n} \in\left[\sigma, R_{1}\right]
$$

and

$$
J^{\prime}\left(z_{n}\right)[(\phi, \widetilde{\psi})]=0, \text { for all }(\phi, \widetilde{\psi}) \in E_{n}
$$

and hence

$$
\left\{\begin{array}{l}
\int_{\Omega} \nabla u_{n} \nabla \widetilde{\psi}=\int_{\Omega} g\left(\widetilde{v}_{n}\right) \widetilde{\psi} \\
\int_{\Omega} \nabla \widetilde{v}_{n} \nabla \phi=\int_{\Omega} f\left(u_{n}\right) \phi
\end{array} \quad, \forall(\phi, \widetilde{\psi}) \in E_{n}\right.
$$

e) Limit $n \rightarrow \infty$ :

By d) we find a sequence $\left(u_{n}, \widetilde{v}_{n}\right) \in E_{n}$ with

$$
J\left(u_{n}, \widetilde{v}_{n}\right) \rightarrow c \in\left[\sigma, R_{1}\right] \text { and } J_{n}^{\prime}\left(u_{n}, \widetilde{v}_{n}\right)=0, \text { in } E_{n},
$$

and by c) we have $\left\|\left(u_{n}, \widetilde{v}_{n}\right)\right\|_{E} \leq c$. Then $\left(u_{n}, \widetilde{v}_{n}\right) \rightarrow(u, \widetilde{v})$ in $E$. Furthermore, we may assume that

$$
\widetilde{v}_{n} \rightarrow \widetilde{v} \text { in } L^{\alpha}, \text { for all } \alpha \geq 1 \text {; }
$$

Indeed, we have for any $\delta>0$

$$
W_{0}^{1} L(2, q) \subset W_{0}^{1} L(2-\delta, 2-\delta)=W_{0}^{1,2-\delta} \subset L^{\frac{(2-\delta) 2}{\delta}},
$$

and hence a compact embedding into $L^{\alpha}$, for all $1 \leq \alpha<\frac{(2-\delta) 2}{\delta}$.

Using (9.5), (9.6) and assumption A3) one concludes now as in [17], Lemma 2.1 , that

$$
\int_{\Omega} f\left(u_{n}\right) \rightarrow \int_{\Omega} f(u), \int_{\Omega} g\left(\widetilde{v}_{n}\right) \rightarrow \int_{\Omega} g(\widetilde{v})
$$

Thus, in (9.14) we can take the limit $n \rightarrow \infty$ to obtain

$$
\left\{\begin{array}{l}
\int_{\Omega} \nabla u \nabla \widetilde{\psi}=\int_{\Omega} g(\widetilde{v}) \widetilde{\psi} \\
\int_{\Omega} \nabla \widetilde{v} \nabla \phi=\int_{\Omega} f(u) \phi
\end{array} \quad, \forall(\phi, \widetilde{\psi}) \in \cup E_{n}=E .\right.
$$


Hence $(u, \widetilde{v}) \in E$ is a (weak) solution of (9.16).

Finally, we prove that $(u, \widetilde{v}) \in E$ is nontrivial. Assume by contradiction that $u=0$, which implies that also $v=0$. Since $g$ is subcritical, we obtain by A5), for all $\delta>0$

$$
|g(t)| \leq c_{\delta} e^{\delta|t|^{q}}, \forall t \in \mathbb{R}
$$

Now we choose $\widetilde{\psi}=\widetilde{v}_{n}$ in the first equation of (9.16), and estimate by Hölder

$$
\left|\int_{\Omega} g\left(\widetilde{v}_{n}\right) \widetilde{v}_{n}\right| \leq c_{\delta}\left\|e^{\delta\left|\widetilde{v}_{n}\right|^{q}}\right\|_{L^{\beta}}\left\|\widetilde{v}_{n}\right\|_{L^{\alpha}} \leq d_{\delta}\left\|\widetilde{v}_{n}\right\|_{L^{\alpha}}
$$

where we have used that $\left\|\nabla \widetilde{v}_{n}\right\| 2, p \leq c$, and hence by Theorem $\mathrm{D}$, subsection 7.2 above, for $\beta>1$ sufficiently small:

$$
\left\|e^{\delta\left|\widetilde{v}_{n}\right|^{q}}\right\|_{L^{\beta}}=\int_{\Omega} e^{\delta \beta\left|\widetilde{v}_{n}\right|^{q}} \leq c
$$

Since $\left\|\widetilde{v}_{n}\right\|_{L^{\alpha}} \rightarrow 0$ by $(9.15)$, we conclude that $\int g\left(\widetilde{v}_{n}\right) \widetilde{v}_{n} \rightarrow 0$ by $(9.17)$, and hence by the first equation in (9.14) that

$$
\int_{\Omega} \nabla u_{n} \nabla \widetilde{v}_{n} \rightarrow 0
$$

This in turn implies, by choosing $\phi=u_{n}$ in the second equation in (9.14), that also $\int_{\Omega} f\left(u_{n}\right) u_{n} \rightarrow 0$. By assumption A2) we now conclude that

$$
\int_{\Omega} F\left(u_{n}\right) \rightarrow 0, \text { and } \int_{\Omega} G\left(u_{n}\right) \rightarrow 0
$$

Finally, by (9.18) and (9.19) we now obtain that $J\left(u_{n}, \widetilde{v}_{n}\right)=\int_{\Omega} \nabla u_{n} \nabla \widetilde{v}_{n}-$ $\int_{\Omega} F\left(u_{n}\right)+G\left(\widetilde{v}_{n}\right) \rightarrow 0$; but this contradicts $(9.12)$, and thus $(u, \widetilde{v}) \neq(0,0)$.

This completes the proof.

\subsection{Critical systems in dimension $N=2$}

For the "critical" system (S2) not much is known. Indeed, we can state the following

\section{Open problems}

- loss of compactness and concentration phenomena for systems with critical growth, i.e. when the exponents lie on the "exponential critical hyperbola"

- non-existence of (radial, positive?) solutions for certain model equations with critical growth

- existence of instantons, or optimal concentrating sequences 
- group invariance and Pohozaev type identities

Concerning existence results for systems in $N=2$ with critical growth, only the following result is known, see [19].

Let $d$ denote the inner radius of the set $\Omega$, that is $d$ is equal to the radius of the largest open ball contained in $\Omega$. Recall that we say that a function $h$ has critical growth at $+\infty$ if there exists $\gamma_{0}>0$, such that

$$
\lim _{t \rightarrow+\infty} \frac{h(t)}{e^{\gamma t^{2}}}=0, \forall \gamma>\gamma_{0}, \text { and } \lim _{t \rightarrow+\infty} \frac{h(t)}{e^{\gamma t^{2}}}=+\infty, \forall \gamma<\gamma_{0} ;
$$

in this case we say that $\gamma_{0}$ is the critical exponent of $h$.

Theorem 9.5 Assume that $f$ and $g$ satisfy assumptions A1) - A3) in subsection 9.2, and that $f$ and $g$ have critical growth with critical exponents $\alpha_{0}$, resp. $\beta_{0}$. Furthermore suppose that

$$
\lim _{t \rightarrow+\infty} \frac{t f(t)}{e^{\alpha_{0} t^{2}}}>\frac{4}{d^{2} \sqrt{\alpha_{0} \beta_{0}}} \text { and } \lim _{t \rightarrow+\infty} \frac{t g(t)}{e^{\beta_{0} t^{2}}}>\frac{4}{d^{2} \sqrt{\alpha_{0} \beta_{0}}} .
$$

Then system $(S 2)$ possesses a nontrivial weak solution $(u, v) \in E$.

Proof. The proof is a combination of the proof of Theorem 3.2 for the scalar equation and the proof of Theorem 9.3 for the subcritical system. We refer the interested reader to [19].

Note that this theorem gives an existence result for two dimensional systems in which both nonlinearities have the same critical growth. This corresponds to the case lying on the diagonal of the exponential critical hyperbola. Existence of solutions for critical cases which are not on the diagonal remains an open problem.

\section{References}

[1] R. A. Adams and J.J. Fournier, Sobolev Spaces, 2nd ed. Academic Press, New York 2003.

[2] Adimurthi, Existence of positive solutions of the semilinear Dirichlet problem with critical growth for the $n$-Laplacian, Ann. Sc. Norm. Sup. Pisa, vol. XVII (1990), p 393-413.

[3] Adimurthi, S.L. Yadava, Multiplicity results for semilinear elliptic equations in a bounded domain of $\mathbb{R}^{2}$ involving criitical exponent, Ann. Sc. Norm. Sup. Pisa XVII (1990), 481-504.

[4] A. Ambrosetti, H. Brezis and G. Cerami, Combined effects of concave and convex nonlinearities in some elliptic problems, J. Funct. Anal., 122 (1994), $519-543$. 
[5] A. Ambrosetti and P. Rabinowitz, Dual variational methods in critical point theory and applications, J. Funct. Anal., 14 (1973), 349-381.

[6] F.V. Atkinson, L.A. Peletier, Ground states and Dirichlet problems for $-\Delta u=f(u)$ in $\mathbb{R}^{2}$, Arch. Rational Mech. Anal. 96 (1986), 147-165.

[7] Th. Aubin, Problèmes isopérimétriques et espace de Sobolev, J. Diff. Geom. 11 (1976), 573-598.

[8] Th. Bartsch, Y. Ding, Deformation theorems on non-metrizable vector spaces and applications to critical point theory, Math. Nachr. 279 (2006), $1267-1288$.

[9] H. Brezis, Laser beams and limiting cases of Sobolev inequalities, Nonlinear Partial Differential Equations and their Applications, Collège de France Seminar, Vol. II, H. Brezis, J.L.Lions ed., Pitman (1982)

[10] H. Brezis, L. Nirenberg Positive solutions of nonlinear elliptic equations involving critical Sobolev exponents, Comm. PUre Appl. Math. 36 (1983), 437-477.

[11] H. Brezis, S. Wainger, A note on limiting cases of Sobolev embeddings and convolution inequalities, Comm. P.D.E. 5 (1980), 773-789.

[12] L. Carleson, A. Chang, On the existence of an extremal function for an inequality of J. Moser, Bull. Sc. Math. 110 (1986), 113-127.

[13] I. Ekeland, R. Temam, Analyse convexe et problèmes variationnels, Paris Gauthier-Villars, 1974

[14] D. de Figueiredo, Lectures on the Ekeland variational principle with applications and détours, TATA Institute, Springer-Verlag, 1989.

[15] D. G. de Figueiredo and P. Felmer, On superquadratic elliptic systems. Trans. Amer. Math. Soc. 343 (1994), 99-116.

[16] D. de Figueiredo and P. L. Lions, On pairs of positive solutions for a class of semilinear elliptic problems, Indiana Univ. Math. J., 34 (1985), 591-606.

[17] D. G. de Figueiredo, O. H. Miyagaki and B. Ruf, Elliptic equations in $\mathbb{R}^{2}$ with nonlinearities in the critical growth range, Calc. Var. 3 (1995), 139153.

[18] D. G. de Figueiredo, J. M. do Ó and B. Ruf, On an inequality by $N$. Trudinger and J. Moser and related elliptic equations, Comm. Pure Appl. Math. 55 (2002), 135-152. 
[19] D.G. de Figueiredo, J.M. do Ó, B. Ruf, Critical and subcritical elliptic systems in dimension two, Indiana University Mathematics Journal, 53, (2004), 1037 - 1053.

[20] D.G. de Figueiredo, J.M. do Ó, B. Ruf, An Orlicz-space approach to superlinear elliptic systems, J. Funct. An. 224 (2005), 471-496.

[21] D. G. de Figueiredo and B. Ruf, On the existence and non-existence of solutions for elliptic equations with critical growth in $R^{2}$, Comm. Pure Appl. Math., 48, (1995), 639-655

[22] D.G. de Figueiredo, B. Ruf, On elliptic systems with nonlinearities of arbitrary growth, Mediterranean J. Math. 1, (2004), 417-431.

[23] D. de Figueiredo, J.- P. Gossez and P. Ubilla, Local superlinearity and sublinearity for indefinite semilinear elliptic problems, J. Funct. Anal., 199 (2003), 452-467.

[24] Flucher, M., Extremal functions for the Trudinger-Moser inequality in 2 dimensions, Comm. Math. Helv. 67 (1992), 471-479.

[25] B. Gidas, W.N. Ni and L. Nirenberg, Symmetry and related properties via maximum principle, Comm. Math. Phys. 68 (1979), 209-243.

[26] J.P. Gossez, Orlicz spaces and strongly nonlinear elliptic problems, Trabalho de Matemática, No. 103, Departamento de Matematica, Universidade de Brasilia, 1976.

[27] N. Ghoussoub, Duality and perturbation methods in critical point theory, Cambridge Tracts in Mathematics, 107, Cambridge University Press, 1993.

[28] J. Hulshof, R.C.A.M. van der Vorst, Differential systems with strongly indefinite variational structure, J. Funct. Anal. 114 (1993), 32-58.

[29] J. Hulshof, R.C.A.M. van der Vorst, Asymptotic behaviour of ground states, Proc. AMS, 124 (1996), 2423-2431.

[30] J. Hulshof, E. Mitidieri, R.C.A.M. van der Vorst, Strongly indefinite systems with critical Sobolev exponents, Trans. AMS, 350, (1998), 23492365 .

[31] O. Kavian, Introduction à la théorie des points critiques, Springer Verlag, 1993

[32] M.A. Krasnoselskiǐ, J.B. Rutickiǐ, Convex Functions and Orlicz Spaces, Transl. first Russian by Leo F. Boron, P. Noordhoff ltd., Groningen, 1961. 
[33] W. Kryszewski, A. Szulkin, Generalized linking theorem with an application to a semilinear Schrdinger equation, Adv. Differential Equations 3 (1998), 441-472.

[34] E. Mitidieri, A Rellich type identity and applications, Commun. PDE 18 (1993), 125-151

[35] S. Li, M. Willem, Applications of local linking to critical point thoery, J. Math anal. Appl. 189 (1995), 6-32.

[36] P.L. Lions, the concentration-compactness principle in the calculus of variations, part I, Rev. Mat. Iberoam. 1 (1985), 145-201

[37] J.L. Lions, E. Magenes, Problèmes aux limites non homogènes et applications: vol. 1,2 , 1968

[38] J. Moser, A sharp form of an inequality by N. Trudinger, Ind. Univ. J. 20 (1971), 1077-1092.

[39] R. Palais and S. Smale, A generalized Morse theory, Bull. Amer. Soc. 70 (1964), 165-171.

[40] S.I. Pohozaev, Eigenfunctions of the equation $\Delta u+\lambda f(u)=0$, Soviet Mat. Dokl. 6 (1965), 1408-1411 (english transl. Dokl. Adad. Nauk. SSRR 165 (1965), 33-36).

[41] S.I. Pohozaev, The Sobolev embedding in the case $p l=n$, Proc. Tech. Sci. Conf. on Adv. Sci. Research 1964-1965, Mathematics Section, Moskov. Ènerget. Inst. Moscow, (1965), 158-170.

[42] P. Pucci and J. Serrin, A general variational identity, Ind. Math. Univ. J. 35 (1986), 681-703

[43] P.H. Rabinowitz, Minimax Methods in Critical Point Theory with Applications to Differential Equations, CBMS Regional Conference Series in Mathematics, vol. 65, American Mathematical Society, Providence, RI, 1986.

[44] M.M. Rao, Z.D. Ren, Theory of Orlicz Spaces, Monographs and Textbooks in Pure and Applied Mathematics, vol. 146, Marcel Dekker, Inc., New York, 1991

[45] Ruf, B., Lorentz spaces and Nonlinear Elliptic systems, Progr. Nonlin. Diff. Equ. 66 (2005), 471-489.

[46] R.S. Strichartz, A note on Trudinger's extension of Sobolev's inequality, Indiana U. Math. J. 21 (1972), 841-842.

[47] M. Struwe, Variational Methods, Springer-Verlag, 1990. 
[48] G. Talenti, Best constant in Sobolev inequality, Ann. Mat. Pura Appl. 110 (1976), 353-372.

[49] N. S. Trudinger, On imbeddings into Orlicz spaces and some applications, J. Math. Mech. 17 (1967), 473-484.

[50] R.C.A.M. van der Vorst, Variational identities and applications to differential systems, Arch. Rational Mech. Anal. 116 (1992), 375-398.

[51] M. Willem, Minimax Theorems, Progress in Nonlinear Differential Equations and their Applications, Birkhäuser, Boston, 1996. 